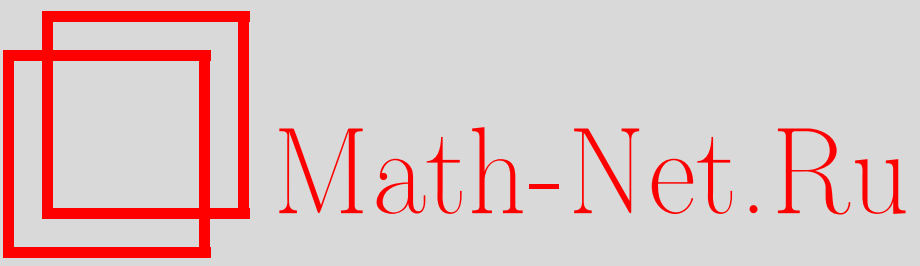

А. А. Боровков, А. А. Могульский, Экспоненциальные неравенства чебышевского типа для сумм случайных векторов и для траекторий случайных блужданий, Теория вероятн. и ее примен., 2011, том 56, выпуск 1, 3-29

DOI: https://doi.org/10.4213/tvp4321

Использование Общероссийского математического портала Math-Net.Ru подразумевает, что вы прочитали и согласны с пользовательским соглашением

http://www.mathnet.ru/rus/agreement

Параметры загрузки:

IP: 35.174 .16 .151

26 апреля 2023 г., 17:44:23 
(c) 2011 г. $\quad$ БОРОВКОВ А. А.* , МОГУЛЬСКИЙ А. А.*

\section{ЭКСПОНЕНЦИАЛЬНЫЕ НЕРАВЕНСТВА ЧЕБЫШЕВСКОГО ТИПА ДЛЯ СУММ СЛУЧАЙНЫХ ВЕКТОРОВ И ДЛЯ ТРАЕКТОРИЙ СЛУЧАЙНЫХ БЛУЖДАНИЙ}

В работе получены аналоги известного экспоненциального неравенства Чебышёва

$$
\mathbf{P}(\xi \geqslant x) \leqslant e^{-\Lambda^{(\xi)}(x)}, \quad x>\mathbf{E} \xi,
$$

для распределения случайной величины $\xi$, где

$$
\Lambda^{(\xi)}(x):=\sup _{\lambda}\left\{\lambda x-\ln \mathbf{E} e^{\lambda \xi}\right\}
$$

есть функция уклонений для $\xi$. Обобщения установлены для многомерных векторов $\xi$, для сумм векторов и для траекторий случайных процессов, ассоциированных с такими суммами.

Ключевые слова и фразы: условие Крамера, функция уклонений, случайное блуждание, функционал уклонений, интеграл уклонений, выпуклое множество, большие уклонения, принцип больших уклонений, расширенный принцип больших уклонений, неравенства для вероятностей больших уклонений.

В $\S 1$ работы получены многомерные аналоги известного экспоненциального неравенства Чебышева

$$
\mathbf{P}(\xi \geqslant x) \leqslant e^{-\Lambda^{(\xi)}(x)}, \quad \Lambda^{(\xi)}(x):=\sup _{\lambda}\left\{\lambda x-\ln \mathbf{E} e^{\lambda \xi}\right\}, \quad x>\mathbf{E} \xi,
$$

для случайной величины $\xi$. В $\S 2$ установлены неравенства для сумм многомерных векторов $\xi$ и траекторий случайных процессов, ассоциированных с такими суммами. В $\S 3$ изучены свойства случайной величины $\gamma=\Lambda^{(\xi)}(\xi)$, играющей важную роль в ряде результатов $\S \S 1,2$.

Результаты работы будут использованы при доказательстве расширенного принципа больших уклонений (см. [1]) для траекторий случайных блужданий (см. [2]).

${ }^{*}$ Институт математики им. С. Л. Соболева СО РАН, пр-т Академика Коптюга, 4, 630090 Новосибирск, Россия; e-mail: borovkov@math.nsc.ru, mogul@math.nsc.ru

1) Работа выполнена при поддержке грантами РФФИ (№ 05-01-00810, 07-01-00595, 08-01-00962), НШ-8980.2006.1, РНШ.2.1.1.1379. 
1. Экспоненциальные неравенства чебышевского типа в многомерном случае.

1.1. Формулировки основных утверждений. Пусть $\xi \in \mathbf{R}^{d}-$ случайный вектор в $d$-мерном пространстве Евклида $\mathbf{R}^{d}$. Скалярное произведение и норму в $\mathbf{R}^{d}$ обозначим $\langle\alpha, \beta\rangle$ и $|\alpha|:=\sqrt{\langle\alpha, \alpha\rangle}$ соответственно. Пусть, далее, $\Lambda(\alpha)=\Lambda^{(\xi)}(\alpha)$ - функция уклонений случайного вектора $\xi$ :

$$
\Lambda(\alpha)=\Lambda^{(\xi)}(\alpha):=\sup _{\lambda}\{\langle\lambda, \alpha\rangle-A(\lambda)\}, \quad \alpha \in \mathbf{R}^{d},
$$

где $A(\lambda)=A^{(\xi)}(\lambda):=\ln \mathbf{E} e^{\langle\lambda, \xi\rangle}, \lambda \in \mathbf{R}^{d}$. Рассмотрим произвольное измеримое множество $B \subset \mathbf{R}^{d}$ и обозначим

$$
\Lambda(B):=\inf _{\alpha \in B} \Lambda(\alpha) .
$$

Если множество $B$ пусто, то мы положим $\Lambda(B)=\infty$. Для произвольного множества $B \subset \mathbf{R}^{d}$ через $(B)$ будем обозначать внутренность $B$, т.е. совокупность таких точек $\alpha$, которые входят в $B$ вместе с некоторой окрестностью, и через $[B]$ - замыкание множества $B$. Для $v \geqslant 0$ рассмотрим множество

$$
\Lambda_{v}:=\{\alpha: \Lambda(\alpha) \leqslant v\},
$$

в точках которого функция уклонений не превосходит $v$. Множество, где функция уклонений конечна, обозначим

$$
\Lambda_{<\infty}:=\bigcup_{v \geqslant 0} \Lambda_{v}=\{\alpha: \Lambda(\alpha)<\infty\} .
$$

Поскольку функция уклонений $\Lambda(\alpha)$ выпукла вниз: для $p \geqslant 0, q \geqslant 0$

$$
\Lambda(p \alpha+q \beta) \leqslant p \Lambda(\alpha)+q \Lambda(\beta),
$$

и полунепрерывна снизу:

$$
\varliminf_{\beta \rightarrow \alpha} \Lambda(\beta) \geqslant \Lambda(\alpha)
$$

(эти свойства легко вытекают из определения (1.1)), то множества $\Lambda_{v}$ выпуклы и замкнуты при любом конечном $v \geqslant 0$, а множество $\Lambda_{<\infty}$ выпукло. Функция уклонений $\Lambda(\alpha)$ обладает следующим характеристическим свойством. Для нормированных сумм $\zeta_{n}:=S_{n} / n, S_{n}:=\xi_{1}+\cdots+\xi_{n}$ (слагаемые $\xi_{k}$ независимы и распределены как $\xi$ ), в силу локального принципа больших уклонений (л.п.б.у.; см., например, [1], [3], [4]) выполняется

$$
\lim _{\varepsilon \rightarrow 0} \lim _{n \rightarrow \infty} n^{-1} \ln \mathbf{P}\left(\zeta_{n} \in(\alpha)_{\varepsilon}\right)=-\Lambda(\alpha),
$$


где $(\alpha)_{\varepsilon}:=\left\{\beta \in \mathbf{R}^{d}:|\alpha-\beta|<\varepsilon\right\}$ есть $\varepsilon$-окрестность точки $\alpha$. Это означает, что при больших $n$ и малых $\varepsilon$ вероятности $\mathbf{P}\left(\zeta_{n} \in(\alpha)_{\varepsilon}\right)$ ведут себя асимптотически как $e^{-n \Lambda(\alpha)+o(n)}$ (точнее, $-n^{-1} \ln \mathbf{P}\left(\zeta_{n} \in(\alpha)_{\varepsilon}\right)$ располагается вблизи значения $\Lambda(\alpha))$.

Предварительно заметим, что в одномерном случае $d=1$ экспоненциальное неравенство Чебышёва для выпуклых множеств $B$ может быть записано в виде

$$
\mathbf{P}(\xi \in B) \leqslant e^{-\Lambda(B)} .
$$

Действительно, если $\Lambda(B)=0$, то неравенство тривиально. Если $\Lambda(B)>0$, то либо $b_{+}:=\inf \{\alpha: \alpha \in B\}>\mathbf{E} \xi$, либо $b_{-}:=\sup \{\alpha: \alpha \in$ $B\}<\mathbf{E} \xi$. Если $b_{+}>\mathbf{E} \xi, b_{+} \in B$, то $\Lambda\left(b_{+}\right)=\Lambda(B)>0$ и (1.4) вытекает из неравенства Чебышёва:

$$
\mathbf{P}(\xi \in B) \leqslant \mathbf{P}\left(\xi \geqslant b_{+}\right) \leqslant e^{-\Lambda\left(b_{+}\right)} .
$$

Если же $\Lambda\left(b_{+}\right)<\Lambda(B)$ (это возможно лишь, если $b_{+} \notin B, \mathbf{P}\left(\xi \leqslant b_{+}\right)=1$ и $\Lambda$ имеет разрыв в точке $\left.b_{+}\right)$, то $\Lambda(B)=\infty, \mathbf{P}(\xi \in B)=0$ и неравенство (1.4) по-прежнему верно. Случай $b_{-}<\mathbf{E} \xi$ рассматривается аналогично. Ясно, что «односторонние» множества $B$ (т.е. лежащие по одну сторону от $\mathbf{E} \xi$ ) допускают вложение в выпуклые множества с теми же границами $b_{ \pm}$и для них также справедливо неравенство (1.4).

Вернемся к общему случаю $d \geqslant 1$. Основные утверждения настоящего параграфа состоят в следующем.

Теорема 1.1. Если $B-$ произвольное выпуклое открытое множество, то справедливо неравенство

$$
\mathbf{P}(\xi \in B) \leqslant e^{-\Lambda(B)} .
$$

Теорема 1.2. Для произвольного множества В справедливо неравенство

$$
\mathbf{P}(\xi \in B) \leqslant e^{-\Lambda\left(\left[B^{\mathrm{con}}\right]\right)},
$$

где $B^{\text {con }}$ - выпуклая оболочка $B$.

Из теорем 1.1, 1.2 следует, что неравенство (1.6) справедливо для выпуклых открытых и выпуклых замкнутых множеств. Таким образом, выделение класса выпуклых множеств и использование функции уклонений позволяет найти единую простую форму (1.4), (1.6) неравенства, которое по-прежнему естественно называть неравенством Чебышёва и которое справедливо в пространствах любой размерности, включая бесконечномерный случай (см. ниже теорему 2.2).

Теоремы 1.1, 1.2 будут следствиями более общего утверждения (теоремы 1.3), в котором используются более широкие условия, обеспечивающие выполнение неравенств вида (1.6). Проверка этих условий в 
задачах большой размерности $d$ может представлять известные трудности. Поэтому в качестве основных результатов мы выделяем более простые теоремы 1.1, 1.2. Эти теоремы достаточны для использования в последующих разделах и в ряде приложений.

Чтобы сформулировать теорему 1.3 , нам понадобится ряд понятий.

Если $\Lambda(B)=0$, то $\Lambda([B])=0$ и все утверждения, приведенные выше и в последующем, будут тривиальными. Поэтому мы везде будем предполагать, что $\Lambda(B)>0$. Это означает, что мы будем предполагать также, что выполнено условие Крамера

$[\mathbf{C}] . \mathbf{E} e^{\langle\lambda, \xi\rangle}<\infty$ в некоторой телесной области значений $\lambda$.

В этом случае множество $\Lambda_{<\infty}$ тоже будет телесным.

Чтобы упростить предварительные пояснения, мы предположим на время, что выполнено следующее более сильное, чем $[\mathbf{C}]$, условие.

$\left[\mathbf{C}_{0}\right] . \mathbf{E} e^{\langle\lambda, \xi\rangle}<\infty$ в окрестности точки $\lambda=0$.

Если выполнено условие $\left[\mathbf{C}_{0}\right]$ (в этом случае, не ограничивая общности, можно считать также, что $\mathbf{E} \xi=0$, «сдвинув» при необходимости множество $B$ ), то $\Lambda(\alpha) \rightarrow \infty$ при $|\alpha| \rightarrow \infty$ и при возрастании $v$ множества $\Lambda_{v}$ образует семейство компактных выпуклых расширяющихся множеств, заполняющих собой множество $\Lambda_{<\infty}$, совпадающее (с точностью до границы) с выпуклой оболочкой $\mathrm{S}$ носителя распределения вектора $\xi$. При приближении границы $\Lambda_{v}$ (с ростом $v$ ) к границе $\Lambda_{<\infty}$ расширение $\Lambda_{v}$ в этом направлении замедляется или останавливается вовсе, так что на каких-то участках границы множеств $\Lambda_{v}$ и $\Lambda_{<\infty}$ могут совпадать. Границы $\partial \Lambda_{v}$ множеств $\Lambda_{v}$ называют поверхностями уровня. Они обладают следующим свойством: при всех $\alpha$ таких, что $\Lambda(\alpha)=v$ (тогда $\left.\alpha \in \partial \Lambda_{v}\right)$, правая часть (1.3) равна одному и тому же значению $v$.

Следует отметить, что поверхности уровня $v$ при достаточно больших $v$ могут иметь «пустоты», т.е. для некоторых направлений е уравнение $\Lambda(t \mathrm{e})=v$ может не иметь решений.

Функция $\Lambda(\alpha)$ непрерывна во внутренности $\left(\Lambda_{<\infty}\right)$ множества $\Lambda_{<\infty}$ (это свойство присуще любой выпуклой, заданной в $\mathbf{R}^{d}$ функции) и полунепрерывна снизу, включая границу. Оказывается, что это последнее свойство можно несколько расширить до непрерывности снизу u изнутри $\Lambda_{<\infty}$ на границе $\partial \Lambda_{<\infty}$ (см. ниже лемму 1.1). В дальнейшем такое расширение нам понадобится для выпукльх полунепрерывных снизу функций $J=J(y) \geqslant 0$, заданных в произвольном линейном метрическом пространстве $(\mathbf{Y}, \rho)$. Поэтому лемму 1.1 мы докажем в общем случае - для функций, обладающих отмеченными выше свойствами в произвольном метрическом пространстве $(\mathbf{Y}, \rho)$. Пусть функция $J=J(y): \mathbf{Y} \rightarrow[0, \infty]$ выпукла и полунепрерывна снизу (последнее означает, что для $J$ выполнено соотношение (1.2)). Так как функция $J$ выпукла вниз, то множества $J_{<\infty}:=\{y \in \mathbf{Y}: J(y)<\infty\}$ и $\left[J_{<\infty}\right]$ будут 
выпуклыми. Для любого множества $B \subset \mathbf{Y}$ обозначим

$$
J(B):=\inf _{y \in B} J(y) .
$$

Лемма 1.1. Пусть функиия $J$ полунепрерывна снизу и выпукла, $y \in\left[J_{<\infty}\right], y_{0} \in J_{<\infty}$. Тогда имеют место следуюшие свойства:

(i) (непрерьвность снизу)

$$
\lim _{\varepsilon \rightarrow 0} J\left((y)_{\varepsilon}\right)=J(y)
$$

(ii) (непрерывность изнутри $J_{<\infty}$ вдоль лучей)

$$
\lim _{p \rightarrow 1, p<1} J\left(p y_{0}+(1-p) y\right)=J(y) .
$$

Следствие 1.1. Если $B-$ выпуклое множество и $J((B))<\infty$, mo

$$
J([B])=J(B)=J((B)) .
$$

Доказательства этих утверждений приведены в п. 1.2.

Вернемся к функции $\Lambda$ в пространстве $\mathbf{R}^{d}$ и сформулируем условия для теоремы 1.3. Пусть сначала $\Lambda(B)<\infty$, т.е. существуют $\alpha \in B$, для которых $\Lambda(\alpha)<\infty$. В этом случае при выполнении условия $\left[\mathbf{C}_{0}\right]$ найдется такое $v=v_{B}$, при котором в силу свойств непрерывности функции $\Lambda$ (отмеченных выше в лемме 1.1) и монотонного возрастания компактов $\Lambda_{v}$ эти компакты впервые коснутся $[B]$. Другими словами, найдется точка $\alpha_{B}$, в которой достигается

$$
\min _{\alpha \in[B]} \Lambda(\alpha)=\Lambda([B])=\Lambda\left(\alpha_{B}\right)=: v_{B} .
$$

Ясно, что точка $\alpha_{B}$ лежит на границах множеств $\Lambda_{v_{B}},[B]$ и эти множества соприкасаются: $\alpha_{B} \in \Lambda_{v_{B}} \cap[B] \neq \varnothing$.

Если выполнено лишь условие $[\mathbf{C}]$, то в случае, когда множество $B$ неограничено, «касание» множеств $\Lambda_{v_{B}}$ и $[B]$ может происходить «на бесконечности».

Если $\Lambda(B)=\infty$, то множество $B$ не пересекается с выпуклым множеством $\Lambda_{<\infty}$.

Обозначение е мы будем использовать для единичного вектора.

О п р е д е л е н и е 1.1. Пусть $\Lambda(B)<\infty$. Тогда множество $B$ будем называть $\Lambda$-отделимьл, если существует гиперплоскость $\Pi_{=}:=$ $\{\alpha:\langle\mathrm{e}, \alpha\rangle=b\}$ (проходящая через точку $\alpha_{B}$, если таковая существует), разделяющая множества $B$ и $\Lambda_{v_{B}}=\left\{\alpha: \Lambda(\alpha) \leqslant v_{B}\right\}$ в следующем смысле:

$$
B \subset \Pi_{>}:=\{\alpha:\langle\mathrm{e}, \alpha\rangle>b\}, \quad \Lambda_{v_{B}} \subset \Pi_{\leqslant}:=\{\alpha:\langle\mathrm{e}, \alpha\rangle \leqslant b\} .
$$

В случае $\Lambda(B)=\infty$ множество $B$ будем называть $\Lambda$-отделимыл, если существует гиперплоскость $\Pi_{=}$, разделяющая множества $B$ и $\Lambda_{<\infty}$ :

$$
B \subset \Pi_{>}, \quad \Lambda_{<\infty} \subset \Pi_{\leqslant} .
$$


Теорема 1.3. Если множество В является $\Lambda$-отделимьл, то

$$
\mathbf{P}(\xi \in B) \leqslant e^{-\Lambda([B])} .
$$

Ясно, что замкнутое множество $B$ при $\Lambda(B)<\infty$ не может быть $\Lambda$-отделимым.

Отметим, что свойство $\Lambda$-отделимости является существенным для утверждения теоремы 1.3 (без этого условия неравенство (1.12), вообще говоря, неверно), но оно является и весьма ограничительным. Какие оценки для $\mathbf{P}(\xi \in B)$ можно получить, если множество $B$ не является $\Lambda$-отделимым?

Если $B \subset \bigcup B_{k}$ вложено в объединение не более чем счетного числа выпуклых множеств $B_{k}$, то из теоремы 1.2 получаем

$$
\mathbf{P}(\xi \in B) \leqslant \sum e^{-\Lambda\left(\left[B_{k}\right]\right)} .
$$

Теоремы 1.2, 1.3 не дают других содержательных оценок. Мы получим несколько иной подход, если рассмотрим случайную величину $\gamma:=\Lambda(\xi)$ и «повторную» функцию уклонений $\Lambda^{(\gamma)}$, т.е. функцию уклонений для случайной величины $\gamma$, равной значению функции уклонений $\Lambda=\Lambda^{(\xi)}(\alpha)$ в точке $\xi$. Свойства случайной величины $\gamma$ и функции уклонений $\Lambda^{(\gamma)}$ исследованы в $\S 3$.

Теорема 1.4. Если $\Lambda(B) \geqslant \mathbf{E} \gamma$, mo

$$
\mathbf{P}(\xi \in B) \leqslant e^{-\Lambda^{(\gamma)}(\Lambda(B))} .
$$

\section{2. Доказательства.}

Д о к а з а т е л ь с т в о л е м м ы 1.1. (i) Свойство (1.8) является следствием свойства полунепрерывности снизу (1.2) для $J$ и соотношения (1.9). Действительно, в силу (1.2)

$$
\varliminf_{\varepsilon \rightarrow 0} J\left((y)_{\varepsilon}\right) \geqslant J(y) .
$$

С другой стороны, выберем $y_{0} \in J_{<\infty}$ и положим $y_{p}:=p y_{0}+(1-p) y$, так что $y=y_{1}$. В силу свойств линейного метрического пространства (см. [5, гл. I, $\S 2$, п. 3, с. 23]) имеем $y_{p} \rightarrow y_{1}$ при $p \rightarrow 1$. Поэтому для каждого $\varepsilon>0$ найдется достаточно близкое к единице $p=p(\varepsilon)<1$ такое, что $y_{p(\varepsilon)} \in(y)_{\varepsilon}$ и $p(\varepsilon) \rightarrow 1$ при $\varepsilon \rightarrow 0$. Поэтому в силу (1.9) выполняется

$$
\varlimsup_{\varepsilon \rightarrow 0} J\left((y)_{\varepsilon}\right) \leqslant \lim _{\varepsilon \rightarrow 0} J\left(y_{p(\varepsilon)}\right)=J(y) .
$$

Свойство (1.8) установлено.

(ii) Если $J(y)=\infty$, то (1.9) вытекает из соотношения (1.2) для $J$. Если же $J(y)<\infty$, то рассмотрим функцию

$$
g(p):=J\left(y_{p}\right), \quad p \in[0,1] .
$$


Функция $J$ выпукла и, следовательно

$$
g(p) \leqslant p J\left(y_{1}\right)+(1-p) J\left(y_{0}\right), \quad \varlimsup_{p \rightarrow 1} g(p) \leqslant J\left(y_{1}\right) .
$$

$\mathrm{C}$ другой стороны, в силу неравенства (1.2) и сходимости $y_{p} \rightarrow y_{1}$ при $p \rightarrow 1$ имеем

$$
\varliminf_{p \rightarrow 0} g(p) \geqslant J\left(y_{1}\right)
$$

Это доказывает (1.9). Лемма 1.1 доказана.

Д ок аз а те ль с тв о сл е д с т в и я 1.1. (i) Заметим предварительно следующее. Так как $B$ и $J_{<\infty}-$ выпуклые множества, то выпуклыми будут также множества $[B]$ и $[B] \cap J_{<\infty}$. По условию $J((B))<\infty$ и, стало быть, множество $(B) \cap J_{<\infty}$ непусто. Возьмем точки $y_{0} \in(B) \cap J_{<\infty}, y_{1} \in[B] \cap J_{<\infty}$ и покажем, что

полуотрезок $\left[y_{0}, y_{1}\right):=\left\{y_{p}:=p y_{1}+(1-p) y_{0} ; p \in[0,1)\right\}$ принадлежит $(B)$.

Обе точки $y_{0}$ и $y_{1}$ принадлежат выпуклому множеству $[B] \cap J_{<\infty}$. Поэтому $y_{p}$ также принадлежит этому множеству при всех $p \in[0,1]$, и чтобы доказать (1.13), надо исключить возможность $y_{p} \in \partial B$ при $p \in[0,1)$. Но если $y_{p} \in \partial B$, то существует последовательность точек $v \rightarrow y_{p}$ такая, что $v \notin[B]$. В этом случае $w:=\left(v-p y_{1}\right) /(1-p) \rightarrow y_{0}$ при $v \rightarrow y_{p}$. Так как $y_{0} \in(B)$, то $w \in(B)$ при $v$ достаточно близких к $y_{p}$. Поскольку $v=p y_{1}+(1-p) w$ и $y_{1} \in[B]$, то $v \in[B]$. Полученное противоречие доказывает (1.13).

(ii) Обратимся теперь непосредственно к доказательству (1.10). Из (1.13) следует неравенство

$$
J((B)) \leqslant J\left(p y_{1}+(1-p) y_{0}\right), \quad 0 \leqslant p<1 .
$$

Так как $y_{0}, y_{1}$ принадлежит $J_{<\infty}$, то в силу утверждения (ii) леммы 1.1 правая часть (1.14) при $p \rightarrow 1$ сходится к $J\left(y_{1}\right)$. Поэтому

$$
J((B)) \leqslant J\left(y_{1}\right) .
$$

Отсюда в силу произвольности $y_{1} \in[B] \cap J_{<\infty}$ получаем неравенство

$$
J((B)) \leqslant J([B])
$$

которое, вместе с очевидными соотношениями

$$
J((B)) \geqslant J(B) \geqslant J([B]),
$$

доказывает (1.10). Следствие 1.1 доказано. 
3 а м е ч а н и е 1.1. В следствии 1.1 условие $J((B))<\infty$ нельзя заменить условием $J(B)<\infty$, о чем свидетельствует (применительно к случаю $\mathbf{Y}=\mathbf{R}^{d}, J=\Lambda$ ) следующий пример.

П р и м е р 1.1. Пусть случайный вектор $\xi \in \mathbf{R}^{2}$ принимает три значения $\beta^{(1)}=(0,2), \beta^{(2)}=(1,1), \beta^{(3)}=(1,-1)$ с вероятностями $p^{(1)}=1 / 2, p^{(2)}=1 / 4, p^{(3)}=1 / 4$ соответственно. В силу л.п.б.у. (1.3) справедливо

$$
\Lambda\left(\beta^{(i)}\right)=-\ln p^{(i)}, \quad i=1,2,3 .
$$

Выберем множество $B=B_{1} \cup\left\{\beta^{(2)}\right\}$, где $B_{1}$ - открытый треугольник с вершинами в точках $\beta^{(1)}, \beta^{(2)}, \beta^{(4)}=(1,2)$. Поскольку $\Lambda(\alpha)=\infty$, если точка $\alpha$ лежит вне замкнутого треугольника с вершинами $\beta^{(1)}, \beta^{(2)}, \beta^{(3)}$, то $\Lambda(B)=-\ln p^{(2)}=\ln 4$. При этом $\Lambda([B]) \leqslant \Lambda\left(\beta^{(1)}\right)=\ln 2$. Таким образом, имеем

$$
\Lambda([B])<\Lambda(B)<\infty .
$$

Равенства (1.10) не выполнены.

Д о к а з а т е ль с т в о т е о р е м ы 1.3. Напомним, что функция уклонений $\Lambda(\cdot)$ содержит всю информацию о распределении величины $\xi$, так как по формуле обращения по $\Lambda(\cdot)$ однозначно восстанавливается функция $A(\cdot)$. В частности, справедливо следующее соотношение, позволяющее выразить «одномерные» функции уклонений через «многомерные».

Лемма 1.2. Для любого $\alpha_{1} \in \mathbf{R}$ справедливо равенство

$$
\Lambda^{\left(\xi_{(1)}\right)}\left(\alpha_{1}\right)=\inf _{\alpha_{2}, \ldots, \alpha_{d}} \Lambda(\alpha)
$$

где $\xi_{(i)}, \alpha_{i}-$ координать векторов $\xi, \alpha$ соответственно. ${ }^{1)}$

Справедливость равенства (1.15) в точках $\alpha_{1}$ непрерывности функции $\Lambda^{\left(\xi_{(1)}\right)}\left(\alpha_{1}\right)$ доказана в $[6$, теорема 16.3$]$.

1) Соотношение (1.15) допускает простую вероятностную интерпретацию с помощью л.п.б.у. (1.3), которая может быть положена в основу «вероятностного» доказательства соотношения (1.15). В соответствии с л.п.б.у. $\Lambda^{\left(\xi_{(1)}\right)}\left(\alpha_{1}\right)$ определяет асимптотику $n^{-1} \ln \mathbf{P}\left(S_{1, n} / n \in\left(\alpha_{1}\right)_{\varepsilon}\right)$ при $n \rightarrow \infty$ и малых $\varepsilon$, где $S_{1, n}$ - первая координата $S_{n}$. Будем считать для удобства изложения в этих пояснениях, что норма $|\alpha|$ определяется соотношением $|\alpha|=\max _{j}\left|\alpha_{j}\right|$. Тогда окрестности $(\alpha]_{\varepsilon}$ (с присоединенными «правыми» $d-1$ гранями, соответствующими координатам $\left.\alpha_{2}, \ldots, \alpha_{d}\right)$ будут «кубиками» и $\mathbf{P}\left(S_{1, n} / n \in\left(\alpha_{1}\right)_{\varepsilon}\right)$ может быть описана также как

$$
\Sigma_{\varepsilon}:=\sum_{k} \mathbf{P}\left(\zeta_{n} \in\left(z_{k}\right]_{\varepsilon}\right),
$$

где $z_{k}$ - центры кубиков из пласта $\left(\alpha_{1}\right)_{\varepsilon}$ таких, что $\bigcup_{k}\left(z_{k}\right]_{\varepsilon}=\left(\alpha_{1}\right)_{\varepsilon}$. Нетрудно видеть, что асимптотику $n^{-1} \ln \Sigma_{\varepsilon}$ будет определять максимальное слагаемое в сумме $\Sigma_{\varepsilon}$, которое в свою очередь в соответствии с л.п.б.у. (1.3) будет определять значение $\min _{k} \Lambda\left(z_{k}\right)$. Это приводит к (1.15). 
Д о к а з а т е л ь с т в о. Обозначим $f\left(\alpha_{1}\right)$ и $g\left(\alpha_{1}\right)$ левую и правую части (1.15) соответственно. Убедимся, что функция $g\left(\alpha_{1}\right)$ выпукла. В силу выпуклости функции уклонений для любых $p \geqslant 0, q \geqslant 0, p+q=1$, $\alpha^{(1)}, \alpha^{(2)} \in \mathbf{R}^{d}$ выполняется

$$
g\left(p \alpha_{1}^{(1)}+q \alpha_{1}^{(2)}\right) \leqslant \Lambda\left(p \alpha^{(1)}+q \alpha^{(2)}\right) \leqslant p \Lambda\left(\alpha^{(1)}\right)+q \Lambda\left(\alpha^{(2)}\right) .
$$

Минимизируя правую часть последнего неравенства по переменным $\alpha_{2}^{(1)}, \ldots, \alpha_{d}^{(1)}$ и $\alpha_{2}^{(2)}, \ldots, \alpha_{d}^{(2)}$, получаем

$$
g\left(p \alpha_{1}^{(1)}+q \alpha_{1}^{(2)}\right) \leqslant p g\left(\alpha_{1}^{(1)}\right)+q g\left(\alpha_{1}^{(2)}\right) .
$$

Выпуклость функции $g\left(\alpha_{1}\right)$ установлена.

Докажем теперь равенство (1.15) для всех точек $\alpha_{1}$, в которых функция $f\left(\alpha_{1}\right)$ непрерывна. Следуя доказательству теоремы 16.3 в [6] и вычисляя преобразование Лежандра $g^{*}$ над функцией $g\left(\alpha_{1}\right)$, получаем

$$
\begin{aligned}
g^{*}\left(\alpha_{1}\right) & :=\sup _{\alpha_{1}}\left\{\lambda_{1} \alpha_{1}-g\left(\alpha_{1}\right)\right\}=\sup _{\alpha_{1}}\left\{\lambda_{1} \alpha_{1}-\inf _{\alpha_{2}, \ldots, \alpha_{d}} \Lambda(\alpha)\right\} \\
& =\sup _{\alpha_{1}} \sup _{\alpha_{2}, \ldots, \alpha_{d}}\left\{\lambda_{1} \alpha_{1}-\Lambda(\alpha)\right\}=A^{(\xi)}\left(\left(\lambda_{1}, 0, \ldots, 0\right)\right)=A^{\left(\xi_{(1)}\right)}\left(\lambda_{1}\right) .
\end{aligned}
$$

Поэтому двойное преобразование $g^{* *}$ над $g$ имеет вид

$$
g^{* *}\left(\alpha_{1}\right)=\sup _{\lambda_{1}}\left\{\lambda_{1} \alpha_{1}-A^{\left(\xi_{(1)}\right)}\left(\lambda_{1}\right)\right\}=\Lambda^{\left(\xi_{(1)}\right)}\left(\alpha_{1}\right)=f\left(\alpha_{1}\right) .
$$

Мы доказали тождество

$$
f\left(\alpha_{1}\right)=g^{* *}\left(\alpha_{1}\right)
$$

Поскольку выпуклая функция $g\left(\alpha_{1}\right)$ может не совпадать с $g^{* *}\left(\alpha_{1}\right)$ только в точках разрывов этих функций (точки разрывов функций $f\left(\alpha_{1}\right), g\left(\alpha_{1}\right)$, $g^{* *}\left(\alpha_{1}\right)$ совпадают), то тождество (1.15) для всех точек непрерывности функций $f\left(\alpha_{1}\right), g\left(\alpha_{1}\right)$ доказано.

Функция $f\left(\alpha_{1}\right)=\Lambda^{\left(\xi_{(1)}\right)}\left(\alpha_{1}\right)$ может иметь лишь две точки разрыва $\alpha_{1 \pm}$, являющиеся границами множества $\Lambda_{<\infty}^{\left(\xi_{(1)}\right)}$. Точки $\alpha_{1 \pm}$ будут точками разрыва лишь в случае, когда $p_{ \pm}:=\mathbf{P}\left(\xi_{(1)}=\alpha_{1 \pm}\right)>0$; при этом $p_{ \pm}=$ $\exp \left\{-\Lambda^{\left(\xi_{(1)}\right)}\left(\alpha_{1 \pm}\right)\right\}$.

Для доказательства равенства $f\left(\alpha_{1 \pm}\right)=g\left(\alpha_{1 \pm}\right)$ покажем сначала, что

$$
f\left(\alpha_{1 \pm}\right) \leqslant g\left(\alpha_{1 \pm}\right)
$$

Для фиксированного $\alpha_{1} \in\left(\alpha_{1-}, \alpha_{1+}\right)$ положим $\alpha_{1, p}=p \alpha_{1}+(1-p) \alpha_{1+}$. Тогда в силу выпуклости функции $g$ имеем

$$
g\left(\alpha_{1, p}\right) \leqslant p g\left(\alpha_{1}\right)+(1-p) g\left(\alpha_{1+}\right), \quad \varlimsup_{p \downarrow 0} g\left(\alpha_{1, p}\right) \leqslant g\left(\alpha_{1+}\right) .
$$


Так как $f\left(\alpha_{1, p}\right)=g\left(\alpha_{1, p}\right)$ и функция $f$ непрерывна изнутри $\left[\alpha_{1-}, \alpha_{1+}\right]$, то

$$
f\left(\alpha_{1+}\right)=\varlimsup_{p \downarrow 0} f\left(\alpha_{1, p}\right)=\varlimsup_{p \downarrow 0} g\left(\alpha_{1, p}\right) \leqslant g\left(\alpha_{1+}\right) .
$$

Проводя аналогичные рассуждения для точки $\alpha_{1-}$, мы получим (1.16).

Докажем теперь обратное неравенство

$$
f\left(\alpha_{1 \pm}\right) \geqslant g\left(\alpha_{1 \pm}\right) .
$$

Для этого рассмотрим «условный» случайный вектор $\xi^{\left(\alpha_{1 \pm}\right)}$ с распределением

$$
\mathbf{P}\left(\xi^{\left(\alpha_{1 \pm}\right)} \in B\right):=\mathbf{P}\left(\xi \in B \mid \xi_{(1)}=\alpha_{1 \pm}\right)=\frac{\mathbf{P}\left(\xi \in B, \xi_{(1)}=\alpha_{1 \pm}\right)}{p_{ \pm}} .
$$

Поскольку

$$
A^{\left(\xi^{\left(\alpha_{1 \pm}\right)}\right)}(\lambda) \leqslant A(\lambda)-\ln p_{ \pm},
$$

то, учитывая равенство $-\ln p_{ \pm}=f\left(\alpha_{1 \pm}\right)$, получаем неравенство

$$
\Lambda^{\left(\xi^{\left(\alpha_{1 \pm}\right)}\right)}(\alpha) \geqslant \Lambda(\alpha)-f\left(\alpha_{1 \pm}\right) .
$$

Поскольку минимум любой функции уклонений равен 0 и функция уклонений, стоящая в левой части неравенства (1.18), равна $\infty$ для всех $\alpha_{1} \neq \alpha_{1 \pm}\left(\right.$ координата $\xi_{(1)}^{\left(\alpha_{1 \pm}\right)}$ равна $\alpha_{1 \pm}$ с вероятностью 1$)$, то получаем

$$
\left.\inf _{\alpha_{2}, \ldots, \alpha_{d}} \Lambda^{\left(\xi^{\left(\alpha_{1 \pm}\right)}\right)}(\alpha)\right|_{\alpha_{1}=\alpha_{1 \pm}}=0 .
$$

Отсюда и из (1.18) получаем (1.17). Равенство $f\left(\alpha_{1}\right)=g\left(\alpha_{1}\right)$ для всех $\alpha_{1} \in \mathbf{R}$ установлено. Лемма 1.2 доказана.

Вернемся к д о к а з а т е л ь с т в у т е о р е м ы 1.3. Обозначим $\gamma=\langle\mathrm{e}, \xi\rangle$. Тогда с помощью ортогонального преобразования соотношение (1.15) можно записать в виде

$$
\Lambda^{(\gamma)}(v)=\inf _{\langle\mathrm{e}, \alpha\rangle=v} \Lambda(\alpha) .
$$

(i) Пусть множество $B$ является $\Lambda$-отделимым и $\Lambda(B)=\infty$. Тогда $\Pi_{>} \cap \Lambda_{<\infty}=\varnothing$ и, стало быть, $\Lambda\left(\Pi_{>}\right)=\infty$. Другими словами, $\Lambda(\alpha)=\infty$ для всех $\alpha \in \Pi_{>}$. Отсюда и из (1.19) следует, что $\Lambda^{(\gamma)}(v)=\infty$ для всех $v>b$ и, значит, $\Lambda^{(\gamma)}((b, \infty))=\infty$.

В силу (1.11) имеем

$$
\mathbf{P}(\xi \in B) \leqslant \mathbf{P}\left(\xi \in \Pi_{>}\right)=\mathbf{P}(\langle\mathrm{e}, \xi\rangle>b)=\mathbf{P}(\gamma>b) .
$$

Так как $\mathbf{P}(\gamma>b)=\lim _{k \rightarrow \infty} \mathbf{P}(\gamma \geqslant b+1 / k)$, то в силу экспоненциального неравенства Чебышёва (1.5)

$$
\mathbf{P}(\gamma>b) \leqslant \lim _{k \rightarrow \infty} e^{-\Lambda^{(\gamma)}(b+1 / k)}=e^{-\Lambda^{(\gamma)}(b+0)},
$$


где $\Lambda^{(\gamma)}(b+0)=\Lambda^{(\gamma)}((b, \infty))=\infty$. Следовательно, $\mathbf{P}(\xi \in B)=0$, и неравенство (1.12) доказано.

(ii) Пусть теперь $\mathbf{P}(B)<\infty$. Из (1.19) получаем

$$
\Lambda^{(\gamma)}((b, \infty))=\inf _{\Pi_{>}} \Lambda(\alpha)=\Lambda\left(\Pi_{>}\right) .
$$

Поэтому вновь в силу неравенств (1.20), (1.21) находим

$$
\mathbf{P}(\xi \in B) \leqslant e^{-\Lambda^{(\gamma)}((b, \infty))}=e^{-\Lambda\left(\Pi_{>}\right)} .
$$

Но множества $\Lambda_{v_{B}}$ и $\Pi_{>}$не пересекаются. Поэтому $\Lambda(\alpha)>v_{B}$ для любого $\alpha \in \Pi_{>}$. Это означает, что $\Lambda\left(\Pi_{>}\right) \geqslant v_{B}$. Неравенство (1.12) установлено. Теорема 1.3 доказана.

Отметим, что если множество $B$ не является $\Lambda$-отделимым, то равенство $\Lambda([B])=\infty$ не влечет за собой, вообще говоря, равенство $\mathbf{P}(\xi \in B)=0$ (напомним, что множество $\Lambda_{<\infty}$ с точностью до границы совпадает с выпуклой оболочкой носителя распределения вектора $\xi)$. На это указывает следующий пример.

П р и м е р 1.2. Пусть $\Gamma$ - единичная сфера в $\mathbf{R}^{d}, d \geqslant 2$, и $B \supset \Gamma-$ ее замкнутая внешность. Тогда $\Lambda([B])=\infty, \mathbf{P}(\xi \in B)=1$.

Д ок аз а т ел ь с т в о т е о р е м ы 1.1. Так как множества $\Lambda_{v}$, $\Lambda_{<\infty}$ выпуклы, то по теореме Хана-Банаха (см., например, [7, гл. III, $\S 2$, п. $5^{\circ}$, с. 137]) открытое выпуклое множество $B$ является $\Lambda$-отделимым, и для него по теореме 1.3 справедливо (1.12). Остается воспользоваться следствием 1.1, в силу которого правая часть (1.12) совпадает с правой частью (1.6). Теорема 1.1 доказана.

Док а з а т е ль с т в о т е о ре мы 1.2. Поскольку $B \subset B^{\text {con }}$, то достаточно убедиться, что (1.7) справедливо для любого выпуклого множества $B=B^{\text {con }}$.

Множество $B_{N}:=\{\alpha \in B:|\alpha| \leqslant N\}$ вместе с $B$ является выпуклым. Выпуклым множеством является также и $\varepsilon$-окрестность $\left(B_{N}\right)_{\varepsilon}$ множества $B_{N}$. Поэтому в силу теоремы 1.1

$$
\mathbf{P}(\xi \in B) \leqslant \mathbf{P}\left(\xi \in\left(B_{N}\right)_{\varepsilon}\right)+P_{N} \leqslant e^{-\Lambda\left(\left(B_{N}\right)_{\varepsilon}\right)}+P_{N}, \quad P_{N}:=\mathbf{P}(|\xi|>N) .
$$

Пусть $\varepsilon>\varepsilon_{k} \rightarrow 0$ при $k \rightarrow \infty$ и $\alpha_{k}$ - последовательность точек из $\left(B_{N}\right)_{\varepsilon_{k}} \subset\left[\left(B_{N}\right)_{\varepsilon}\right]$ такая, что $\Lambda\left(\alpha_{k}\right) \leqslant \Lambda\left(\left(B_{N}\right)_{\varepsilon_{k}}\right)+1 / k$. Считая, не ограничивая общности, что $\alpha_{k}$ при $k \rightarrow \infty$ сходится к $\alpha_{0} \in\left[B_{N}\right]$, получим в силу полунепрерывности снизу функции $\Lambda$

$$
\begin{aligned}
\mathbf{P}(\xi \in B) & \leqslant \varlimsup_{k \rightarrow \infty} e^{-\Lambda\left(\left(B_{N}\right)_{\varepsilon_{k}}\right)}+P_{N} \leqslant \varlimsup_{k \rightarrow \infty} e^{-\Lambda\left(\alpha_{k}\right)+1 / k}+P_{N} \\
& \leqslant e^{-\Lambda\left(\alpha_{0}\right)}+P_{N} \leqslant e^{-\Lambda\left(\left[B_{N}\right]\right)}+P_{N} .
\end{aligned}
$$

Поскольку $\Lambda\left(\left[B_{N}\right]\right) \downarrow \Lambda([B])$ при $N \uparrow \infty$, то, переходя в $(1.22)$ к пределу при $N \uparrow \infty$, получаем (1.12). Теорема 1.2 доказана. 
Д ок аз а т е л ь с т в о т е о р е м ы 1.4. Таккак $B \subset\{\alpha: \Lambda(\alpha) \geqslant$ $\Lambda(B)\}$, то при $\Lambda(B) \geqslant \mathbf{E} \gamma$

$$
\mathbf{P}(\xi \in B) \leqslant \mathbf{P}(\Lambda(\xi) \geqslant \Lambda(B))=\mathbf{P}(\gamma \geqslant \Lambda(B)) \leqslant e^{-\Lambda^{(\gamma)}(\Lambda(B))} .
$$

Теорема доказана.

2. Экспоненциальные неравенства чебышевского типа для сумм случайных векторов и для траекторий случайных блужданий.

2.1. Экспоненциальные неравенства чебышевского типа для сумм случайных векторов. Пусть $\xi, \xi_{1}, \xi_{2}, \ldots$ - последовательность независимых одинаково распределенных $d$-мерных случайных векторов,

$$
S_{n}:=\sum_{i=1}^{n} \xi_{i}, \quad n \geqslant 1
$$

Положим

$$
\zeta_{n}:=\frac{S_{n}}{n}, \quad A^{\left(\zeta_{n}\right)}(\lambda):=\ln \mathbf{E} e^{\left\langle\lambda, \zeta_{n}\right\rangle}=n A\left(\frac{\lambda}{n}\right) .
$$

Тогда функция уклонений $\Lambda^{\left(\zeta_{n}\right)}(\alpha)$ для $\zeta_{n}$ равна

$$
\Lambda^{\left(\zeta_{n}\right)}(\alpha)=\sup _{\lambda}\left\{\langle\lambda, \alpha\rangle-n A\left(\frac{\lambda}{n}\right)\right\}=n \sup _{\lambda}\left\{\left\langle\frac{\lambda}{n}, \alpha\right\rangle-A\left(\frac{\lambda}{n}\right)\right\}=n \Lambda(\alpha) .
$$

Поэтому непосредственно из теорем 1.1, 1.2 вытекает следующее утверждение.

Следствие 2.1. (i) Для произвольного открытого выпуклого множества В справедливо неравенство

$$
\mathbf{P}\left(\zeta_{n} \in B\right) \leqslant e^{-n \Lambda(B)} .
$$

(ii) Для произвольного выпуклого множества В справедливо неравенство

$$
\mathbf{P}\left(\zeta_{n} \in B\right) \leqslant e^{-n \Lambda\left(\left[B^{\mathrm{con}}\right]\right)},
$$

где $B^{\text {con }}$ - выпуклая оболочка $B$.

Из принципа больших уклонений для $\zeta_{n}$ следует, что оценка $(2.2)$ «экспоненциально» неулучшаема. Для множеств $B \subset B_{v}:=\{\alpha: \Lambda(\alpha) \geqslant$ $v\}$ в [8] установлена оценка

$$
\mathbf{P}\left(\zeta_{n} \in B\right) \leqslant \mathbf{P}\left(\zeta_{n} \in B_{v}\right) \leqslant c n^{(d-1) / 2} e^{-n \Lambda\left(B_{v}\right)}\left(1+\varepsilon_{n}\right),
$$

где $\Lambda\left(B_{v}\right)=v, \varepsilon_{n} \rightarrow 0$ при $n \rightarrow \infty$, постоянная $c$ зависит от распределения случайного вектора $\xi$ и от размерности $d\left(c=2, \varepsilon_{n}=0\right.$ при $d=1)$. 
Интегро-локальные предельные теоремы о точной асимптотике вероятности попадания $S_{n}$ в малый куб в так называемой крамеровской зоне больших уклонений позволяют установить, что для множеств $B_{v}$ справедливо при $n \rightarrow \infty$ представление

$$
\mathbf{P}\left(\zeta_{n} \in B_{v}\right)=c^{\prime} n^{d / 2-1} e^{-n v}\left(1+\varepsilon_{n}^{\prime}\right),
$$

где $\varepsilon_{n}^{\prime} \rightarrow 0$ при $n \rightarrow \infty, c^{\prime}$ зависит от распределения векторов $\xi$ и размерности $d$.

Это означает, что погрешность оценки (2.3) имеет при $n \rightarrow \infty$ порядок роста $n^{1 / 2}$. Порядок роста «минимальной» погрешности неравенства (2.2) (для наиболее широких выпукльхх множеств $B=\Pi_{\geqslant}$) также составляет $n^{1 / 2}$.

Следует отметить, что оценки (2.3), (2.4) являются асимптотическими, при этом явный вид поправочных членов $\varepsilon_{n}, \varepsilon_{n}^{\prime}$ изучен недостаточно. Столь же хороших точных оценок для $\mathbf{P}\left(\zeta_{n} \in B\right)$ с произвольными множествами $B$ получить не удается. Однако можно привести следующее утверждение.

Следствие 2.2. Если $\Lambda(B) \geqslant \mathbf{E} \gamma$, mo

$$
\mathbf{P}\left(\zeta_{n} \in B\right) \leqslant e^{-n \Lambda^{(\gamma)}(\Lambda(B))} .
$$

Неравенство (2.5) не является «экспоненциально» неулучшаемым из-за «потерь» в первом неравенстве в (2.6) (см. ниже). Об этом говорит также неравенство $\Lambda^{(\gamma)}(v)<v-\mathbf{E} \gamma$ (см. (3.8) ниже), справедливое для неограниченных сверху величин $\xi$. Однако при больших $v$ выполняется $\Lambda^{(\gamma)}(v) \sim v$ и неравенства $(2.2),(2.5)$ в известном смысле сближаются $\left(\Lambda^{(\gamma)}(\Lambda(B)) \sim \Lambda(B)\right.$ при больших $\left.\Lambda(B)\right)$.

Д ок а з а т ел ь с т в о с ле д с т в и я 2.2. В силу выпуклости функции уклонений $\Lambda(\alpha)$ имеем

$$
n \Lambda\left(\zeta_{n}\right) \leqslant \sum_{k=1}^{n} \gamma_{k}, \quad \gamma_{k}:=\Lambda\left(\xi_{k}\right) .
$$

Так как $B \subset\{\alpha: \Lambda(\alpha) \geqslant \Lambda(B)\}$, то

$$
\left\{\zeta_{n} \in B\right\} \subset\left\{\Lambda\left(\zeta_{n}\right) \geqslant \Lambda(B)\right\} \subset\left\{\sum_{k=1}^{n} \gamma_{k} \geqslant n \Lambda(B)\right\} .
$$

Из этого соотношения при $\Lambda(B) \geqslant \mathbf{E} \gamma$ получаем в силу экспоненциального неравенства Чебышёва

$$
\mathbf{P}\left(\zeta_{n} \in B\right) \leqslant \mathbf{P}\left(\sum_{k=1}^{n} \gamma_{k} \geqslant n \Lambda(B)\right) \leqslant e^{-n \Lambda^{(\gamma)}(\Lambda(B))} .
$$

Следствие 2.2 доказано. 
2.2. Экспоненциальные неравенства чебышевского типа для траекторий случайных блужданий. Рассмотрим непрерывные случайные ломаные $s_{n}=s_{n}(t), t \in[0,1]$, построенные по узловым точкам $\left(k / n, S_{k} / n\right)$, где $S_{0}=0, k=0,1, \ldots, n$. Траектории $s_{n}(t)$ будут рассматриваться как элементы метрического пространства $(\mathbf{C}, \rho)$, где $\mathbf{C}=\mathbf{C}^{d}[0,1]$ - пространство непрерывных функций $f=f(t)$ на отрезке $[0,1]$ со значениями в $\mathbf{R}^{d}$, с «равномерной» метрикой

$$
\rho(f, g):=\max _{0 \leqslant t \leqslant 1}|f(t)-g(t)| .
$$

Как и прежде, мы будем изучать возможность оценивания сверху вероятности $\mathbf{P}\left(s_{n} \in B\right)$. Важную роль в этой задаче играет интеграл Лебега

$$
I(f):=\int_{0}^{1} \Lambda\left(f^{\prime}(t)\right) d t
$$

определяемый на пространстве абсолютно непрерывных функций $\mathbf{C}_{\mathrm{a}}$ и называемый интегралом уклонений (см. [1], [2]). Здесь $f^{\prime}=f^{\prime}(t)$ обозначает производную абсолютно непрерывной функции

$$
f(t)=\left(f_{(1)}(t), \ldots, f_{(d)}(t)\right) \in \mathbf{C}_{\mathrm{a}}: \quad f^{\prime}(t)=\left(f_{(1)}^{\prime}(t), \ldots, f_{(d)}^{\prime}(t)\right), \quad t \in[0,1] .
$$

Функционал $I$ позволяет с помощью принципа больших уклонений описывать асимптотическое поведение последовательности $\ln \mathbf{P}\left(s_{n} \in(f)_{\varepsilon}\right)$ при малых $\varepsilon>0$ и $n \rightarrow \infty$. Для формулировки этого принципа, который содержится в [9], [10] (близкие результаты имеются в [11]), нам наряду с условием $\left[\mathbf{C}_{0}\right]$ понадобится условие Крамера в его наиболее сильной форме.

$\left[\mathbf{C}_{\infty}\right] . \mathbf{E} e^{\langle\lambda, \xi\rangle}<\infty \partial л я$ всех $\lambda$.

Положим

$$
I_{\mathrm{a}}(B):=\inf \left\{I(f): f \in B \cap \mathbf{C}_{a}, f(0)=0\right\},
$$

где нижнюю грань по пустому множеству считаем равной $\infty$.

Теорема 2.1. Пусть выполнено условие $\left[\mathbf{C}_{\infty}\right]$. Тогда:

(i) (приниип больших уклонений) для любого борелевского множества $B \subset \mathbf{C}$

$$
\begin{aligned}
& \varlimsup_{n \rightarrow \infty} \frac{1}{n} \ln \mathbf{P}\left(s_{n} \in B\right) \leqslant-I_{\mathrm{a}}([B]), \\
& \varliminf_{n \rightarrow \infty} \frac{1}{n} \ln \mathbf{P}\left(s_{n} \in B\right) \geqslant-I_{\mathrm{a}}((B)) ;
\end{aligned}
$$

(ii) (локальньй принцип больших уклонений) для любой функции $f \in \mathbf{C}_{\mathrm{a}}, \quad f(0)=0$

$$
\lim _{\varepsilon \rightarrow 0} \lim _{n \rightarrow \infty} \frac{1}{n} \ln \mathbf{P}\left(s_{n} \in(f)_{\varepsilon}\right)=-I(f) .
$$


Если $f \in \mathbf{C} \backslash \mathbf{C}_{\text {а }}$ или $f(0) \neq 0$, то правую часть в (2.9) следует положить равной $-\infty$.

Утверждения (2.8), (2.9) теоремы 2.1 будут полезны для сравнения с последующими результатами работы.

В [2] показано, что условие $\left[\mathbf{C}_{\infty}\right]$ для утверждений $(2.8),(2.9)$ является излишним. Отчасти на это будет указывать следствие 2.3 , приведенное ниже.

Основным утверждением в этом пункте является следующая теорема.

Теорема 2.2. (i) Для любого открытого выпуклого множества $B \subset \mathbf{C}$ справедливо неравенство

$$
\mathbf{P}\left(s_{n} \in B\right) \leqslant e^{-n I_{\mathrm{a}}(B)} .
$$

(ii) Для любого борелевского множества $B \subset \mathbf{C}$

$$
\mathbf{P}\left(s_{n} \in B\right) \leqslant e^{-n I_{\mathrm{a}}\left(\left[B^{\mathrm{con}}\right]\right)},
$$

где $B^{\text {con }}$ - выпуклая оболочка множества В.

(iii) Если $B-$ борелевское множество, $I_{\mathrm{a}}(B) \geqslant \mathbf{E} \gamma, \gamma=\Lambda(\xi)$, то

$$
\mathbf{P}\left(s_{n} \in B\right) \leqslant e^{-n \Lambda^{(\gamma)}\left(I_{\mathrm{a}}(B)\right)} .
$$

В связи с теоремой 2.2 и некоторыми последующими утверждениями оказывается полезной следующая лемма. mo

Лемма 2.1. Если $B \subset \mathbf{C}-$ выпуклое множество, $I_{\mathrm{a}}((B))<\infty$,

$$
I_{\mathrm{a}}((B))=I_{\mathrm{a}}(B)=I_{\mathrm{a}}([B]) .
$$

Д ок аз а т е л ь с т в. Рассмотрим вспомогательный функционал

$$
I^{\lim }(f):=\lim _{\varepsilon \rightarrow 0} I_{\mathrm{a}}\left((f)_{\varepsilon}\right),
$$

определенный на всех элементах $f \in \mathbf{C}$. Функционал $I^{\lim }$ совпадает с интегралом уклонений $J$, определенным в [12] (см. определение 2.1 и следствие 4.1 в [12]), хотя по форме определения этих функционалов различны.

Нам понадобится следующая лемма.

Лемма 2.2. Функиионал $I^{\lim }(f)$ является выпукльцм и полунепрерьввным снизу.

Д ок аз а т ель с т в о. (i) Убедимся сначала, что функционал $I^{\lim }$ является выпуклым. Для $p \geqslant 0, q \geqslant 0, p+q=1, f, g \in \mathbf{C}$ найдутся последовательности $\left(f_{n}\right)$ и $\left(g_{n}\right)$ такие, что $f_{n} \rightarrow f$ и $g_{n} \rightarrow g$ при $n \rightarrow \infty$ и

$$
p I^{\lim }(f)+q I^{\lim }(g)=\lim _{n \rightarrow \infty}\left(p I\left(f_{n}\right)+q I\left(g_{n}\right)\right)
$$


В силу выпуклости $I(f)$ имеем $p I\left(f_{n}\right)+q I\left(g_{n}\right) \geqslant I\left(p f_{n}+q g_{n}\right)$. Поэтому

$$
p I^{\lim }(f)+q I^{\lim }(g) \geqslant \varlimsup_{n \rightarrow \infty} I\left(p f_{n}+q g_{n}\right) .
$$

Далее, для любого $\varepsilon>0$ и всех достаточно больших $n$ выполняется $p f_{n}+q g_{n} \in(p f+q g)_{\varepsilon}$. Поэтому

$$
I\left(p f_{n}+q g_{n}\right) \geqslant I\left((p f+q g)_{\varepsilon}\right), \quad p I^{\lim }(f)+q I^{\lim }(g) \geqslant I_{\mathrm{a}}\left((p f+q g)_{\varepsilon}\right) .
$$

Переходя к пределу в правой части последнего неравенства при $\varepsilon \rightarrow 0$, получаем

$$
p I^{\lim }(f)+q I^{\lim }(g) \geqslant I^{\lim }(p f+q g) .
$$

Выпуклость функционала $I^{\lim }(f)$ установлена.

(ii) Докажем теперь полунепрерывность $I^{\lim }(f)$ снизу. Возьмем произвольную последовательность $f_{n}$, сходящуюся к $f$ при $n \rightarrow \infty$. Для любого фиксированного $\varepsilon>0$ найдется $N=N_{\varepsilon}<\infty$ такое, что для любых $\delta \in(0, \varepsilon), n \geqslant N$ выполняется

$$
\left(f_{n}\right)_{\delta} \subset(f)_{2 \varepsilon}, \quad I_{a}\left(\left(f_{n}\right)_{\delta}\right) \geqslant I_{\mathrm{a}}\left((f)_{2 \varepsilon}\right) .
$$

Поэтому для $n \geqslant N$

$$
I^{\lim }\left(f_{n}\right)=\lim _{\delta \rightarrow 0} I_{\mathrm{a}}\left(\left(f_{n}\right)_{\delta}\right) \geqslant I_{\mathrm{a}}\left((f)_{2 \varepsilon}\right), \quad \varliminf_{n \rightarrow \infty} I^{\lim }\left(f_{n}\right) \geqslant I_{\mathrm{a}}\left((f)_{2 \varepsilon}\right) .
$$

Переходя в правой части последнего неравенства к пределу при $\varepsilon \rightarrow 0$, получаем

$$
\varliminf_{n \rightarrow \infty} I^{\lim }\left(f_{n}\right) \geqslant I^{\lim }(f) .
$$

Полунепрерывность снизу функционала $I^{\lim }(f)$, а вместе с ней и лемма 2.2 доказаны.

Вернемся к доказательству леммы 2.1. В силу следствия 1.1 для выпуклого и полунепрерывного снизу функционала

$$
J(f)=I^{\lim }(f)
$$

в $\mathbf{C}$ имеют место равенства

$$
J((B))=J(B)=J([B]),
$$

в которых $J(B)$ для любого множества $B \subset \mathbf{C}$ определяется соотношением

$$
J(B):=\inf _{f \in B} J(f) .
$$

Докажем теперь (2.13). Так как для $f \in \mathbf{C}_{\text {а }}$ выполняется $I(f) \geqslant J(f)$ и для любого $B \subset \mathbf{C}$

$$
I_{\mathrm{a}}(B)=\inf \left\{I(f): f \in B \cap \mathbf{C}_{\mathrm{a}}, f(0)=0\right\},
$$


TO

$$
I_{\mathrm{a}}((B)) \geqslant I_{\mathrm{a}}(B) \geqslant I_{\mathrm{a}}([B]) \geqslant J([B]) .
$$

Покажем, что

$$
J((B)) \geqslant I_{\mathrm{a}}((B)) .
$$

Для любого $f \in(B)$ и любого достаточно малого $\varepsilon>0$ выполняется

$$
(f)_{\varepsilon} \subset(B), \quad I_{\mathrm{a}}\left((f)_{\varepsilon}\right) \geqslant I_{\mathrm{a}}((B)) .
$$

Поэтому

$$
J(f)=I^{\lim }(f)=\lim _{\varepsilon \rightarrow 0} I_{\mathrm{a}}\left((f)_{\varepsilon}\right) \geqslant I_{\mathrm{a}}((B)), \quad f \in(B) .
$$

Отсюда следует (2.16). Из соотношений (2.14)-(2.16) вытекает (2.13). Лемма 2.1 доказана.

Отметим, что если выполнено условие $\left[\mathbf{C}_{\infty}\right]$, то, как показано в $[9],[10], I^{\lim }(f)=\infty$ при $f \in \mathbf{C} \backslash \mathbf{C}_{\mathrm{a}}$. Поскольку $I^{\lim }(f)=I(f)$ при $f \in \mathbf{C}_{\mathrm{a}}$, то в этом случае требуемое свойство выпуклости и полунепрерывности снизу функционала $I^{\lim }(f)$ на $\mathbf{C}$ является очевидным следствием свойств $I$ на $\mathbf{C}_{\text {a }}$ и, стало быть, утверждение леммы 2.1 немедленно вытекает из следствия 1.1.

Из теоремы 2.2 и леммы 2.1 вытекает следующее утверждение.

Следствие 2.3. Пусть $B \subset \mathbf{C}$ - вылуклое множество. Тогда:

(i) имеет место неравенство

$$
\mathbf{P}\left(s_{n} \in B\right) \leqslant e^{-n I_{\mathrm{a}}([B])}
$$

(ii) если дополнительно вьполнено хотя бъ одно из следующих условий:

а) множество В является открыттьлм,

b) множество $B$ является замкнутыл,

c) $I_{a}((B))<\infty$ то $[B]$ в правой части (2.17) можно заменить на $B$;

(iii) если В удовлетворяет условиям а) или с) утверждения (ii) $и$ вьголнено соотношение (2.8), то существует предел

$$
\lim _{n \rightarrow \infty} \frac{1}{n} \ln \mathbf{P}\left(s_{n} \in B\right)=-I_{\mathrm{a}}(B) .
$$

В [2] показано, что соотношение (2.18) справедливо всегда (условие (2.8) является излишним).

Для выпуклых множеств $B$ неравенство (2.10) (см. утверждение (i) следствия 2.3) существенно уточняет и усиливает оценку сверху (2.7) в теореме 2.1 сразу в нескольких направлениях: (а) неравенство (2.10) 
является точным неравенством, а не асимптотическим; (b) оно не требует выполнения условия $\left[\mathbf{C}_{\infty}\right] ;(\mathrm{c})$ в его правой части отсутствует операция замыкания множества $B$. Неравенства $(2.10),(2.11)$ могут использоваться также и для доказательства усиленной версии самой теоремы 2.1 (cM. [2]).

Соотношение (2.18) показывает, что «экспоненциальная часть» неравенства (2.10) неулучшаема.

Неравенство (2.12) также уточняет (в известном смысле) оценку (2.7), так как эта оценка, например, для множеств $B_{v}=\{f: I(f) \geqslant v\}$ вообще теряет смысл, поскольку $\left[B_{v}\right]$ совпадает с $\mathbf{C}$ и оценка $(2.7)$ становится тривиальной. В то же время (2.12) при $v>\mathbf{E} \gamma$ дает содержательную экспоненциально неулучшаемую оценку для $\mathbf{P}\left(s_{n} \in B_{v}\right)$ (см. ниже).

Совпадение $\left[B_{v}\right]$ с $\mathbf{C}$ вытекает из того, что для любых $u, \varepsilon$ и $f \in \mathbf{C}$ легко построить такую «быстроколеблющуюся» функцию $g$, что $\rho(f, g)<\varepsilon$, а ее вариация $\operatorname{Var} g:=\int_{0}^{1}\left|g^{\prime}(t)\right| d t \geqslant u$ может быть сделана сколь угодно большой. Поскольку при выполнении условия $\left[\mathbf{C}_{0}\right]$ для некоторых $c_{1}>0, c_{2}<\infty$ и всех $\alpha \in \mathbf{R}^{d}$ выполняется неравенство $\Lambda(\alpha) \geqslant c_{1}|\alpha|-c_{2}$, то $I(g) \geqslant c_{1} u-c_{2}$. Таким образом, для заданного $v>0$ мы можем построить функцию $g$, сколь угодно близкую к $f$ и такую, что $I(g) \geqslant v\left(g \in B_{v}\right.$ при $\left.u \geqslant\left(v+c_{2}\right) / c_{1}\right)$.

Д ок а з а те ль с т в о те о р е м ы 2.2. (i) Обозначим $\mathbf{L}_{n}$ подкласс пространства $\mathbf{C}$, состоящий из выходящих из нуля непрерывных ломаных $l=l(t)$, линейных на каждом интервале вида $((i-1) / n, i / n)$ для $i=1, \ldots, n$. Например, случайная ломаная $s_{n}$ является элементом пространства $\mathbf{L}_{n}$.

Рассмотрим отображение $H: \mathbf{L}_{n} \rightarrow \mathbf{R}^{d n}$, которое каждой ломаной $l \in \mathbf{L}_{n}$ ставит в соответствие вектор $H l=\vec{h}:=\left(h_{1}, \ldots, h_{n}\right)$, где $h_{i}:=n(l((i+1) / n)-l(i / n)), i=1, \ldots, n$. Обратное отображение $H^{(-1)}$ по вектору приращений $\vec{h} \in \mathbf{R}^{d n}$ восстанавливает ломаную в $\mathbf{L}_{n}$ с узлами $\left(i / n, H_{i} / n\right)$, где $H_{i}=h_{1}+\cdots+h_{i}$ при $i=1, \ldots, n$. Очевидно, что $H$ есть взаимно однозначное линейное непрерывное (при «естественной» равномерной метрике $\rho$ в $\mathbf{L}_{n}$ и евклидовой норме в $\left.\mathbf{R}^{d n}\right)$ отображение пространства $\mathbf{L}_{n}$ в пространство $\mathbf{R}^{d n}$. Это отображение ставит в соответствие случайной ломаной $s_{n}$ случайный вектор $H s_{n}=\vec{\xi}:=\left(\xi_{1}, \ldots, \xi_{n}\right)$. При этом отображении образ $H\left(B \cap \mathbf{L}_{n}\right)$ для любого открытого выпуклого множества $B \subset \mathbf{C}$ будет открытым и выпуклым в пространстве $\mathbf{R}^{d n}$. Поэтому в силу теоремы 1.1 в пространстве размерности $d n$ имеем

$\mathbf{P}\left(s_{n} \in B\right)=\mathbf{P}\left(s_{n} \in B \cap \mathbf{L}_{n}\right)=\mathbf{P}\left(\vec{\xi} \in H\left(B \cap \mathbf{L}_{n}\right)\right) \leqslant \exp \left\{-\Lambda^{(\vec{\xi})}\left(H\left(B \cap \mathbf{L}_{n}\right)\right)\right\}$

Заметим, далее, что для любого $\vec{h} \in \mathbf{R}^{d n}$ функция уклонений $\Lambda^{(\vec{\xi})}(\vec{h})$ 
случайного вектора $\vec{\xi}$ в силу независимости координат имеет вид

$$
\Lambda^{(\vec{\xi})}(\vec{h})=\sum_{k=1}^{n} \Lambda\left(h_{k}\right)=n \int_{0}^{1} \Lambda\left(l^{\prime}(t)\right) d t=n I(l),
$$

где $l=H^{(-1)} \vec{h}, \Lambda(\alpha)=\Lambda^{(\xi)}(\alpha)$. Иначе говоря, для $l \in \mathbf{L}_{n}$ и $\vec{h}=H l$ выполняется равенство $\Lambda^{(\vec{\xi})}(\vec{h})=\Lambda^{(\vec{\xi})}(H l)=n I(l)$. Поэтому, при очевидном толковании обозначений, имеем

$$
\Lambda^{(\vec{\xi})}\left(H\left(B \cap \mathbf{L}_{n}\right)\right)=n I_{\mathrm{a}}\left(B \cap \mathbf{L}_{n}\right) \geqslant n I_{\mathrm{a}}(B) .
$$

Это означает, что правая часть в $(2.19)$ не больше, чем $e^{-n I_{\mathrm{a}}(B)}$. Утверждение (i) теоремы 2.2 доказано.

Утверждение (ii) доказывается аналогично теореме 1.2.

Доказательство утверждения (iii) по существу повторяет доказательство теоремы 1.4. Так как $B \cap \mathbf{C}_{\mathrm{a}} \subset\left\{f: I(f) \geqslant I_{\mathrm{a}}(B)\right\}$, то для $I_{\mathrm{a}}(B) \geqslant \mathbf{E} \gamma$ имеем

$$
\begin{aligned}
\mathbf{P}\left(s_{n} \in B\right) & =\mathbf{P}\left(s_{n} \in B \cap \mathbf{C}_{\mathrm{a}}\right) \leqslant \mathbf{P}\left(I\left(s_{n}\right) \geqslant I_{\mathrm{a}}(B)\right) \\
& =\mathbf{P}\left(\int_{0}^{1} s_{n}^{\prime}(t) d t \geqslant I_{\mathrm{a}}(B)\right) \\
& =\mathbf{P}\left(\sum_{k=1}^{n} \Lambda\left(\xi_{k}\right) \geqslant n I_{a}(B)\right)=\mathbf{P}\left(\sum_{k=1}^{n} \gamma_{k} \geqslant n I_{\mathrm{a}}(B)\right) \\
& \leqslant e^{-n \Lambda^{(\gamma)}\left(I_{\mathrm{a}}(B)\right)} .
\end{aligned}
$$

Это доказывает (2.12). Теорема 2.2 доказана.

Отметим, что доказательство неравенства (2.12) не содержит «потерь» (ср. с доказательством следствия 2.2) и, стало быть, оценка (2.12) для вероятностей попадания в множества $B_{v}$, определяемые поверхностями уровня $v$, в бесконечномерном случае является экспоненциально неулучшаемой (в отличие от неравенства (2.5) в конечномерном случае). На неулучшаемость показателя экспоненты в (2.12) указывает также логарифмическая асимптотика вероятности $\mathbf{P}\left(s_{n} \in B_{v}\right)=\mathbf{P}\left(\sum_{k=1}^{n} \gamma_{k} \geqslant\right.$ $n v)$, оцениваемой в (2.20) для множеств $B_{v}=\{f: I(f) \geqslant v\}, v=I_{\mathrm{a}}(B)$. Согласно принципу больших уклонений (см., например, [3]),

$$
\ln \mathbf{P}\left(\sum_{k=1}^{n} \gamma_{k} \geqslant n v\right) \sim-n \Lambda^{(\gamma)}(v) \quad \text { при } \quad n \rightarrow \infty .
$$

Можно добавить также, что $\mathbf{P}\left(s_{n} \in B_{v}\right) \rightarrow 1$ при любом $v<\mathbf{E} \gamma$, а при значениях $v \in\left[\mathbf{E} \gamma, v_{+}\right)$, где $v_{+}$известно, точная асимптотика этой вероятности может быть найдена в явном виде (см., например, [3]).

Так как $\Lambda^{(\gamma)}(v)<v-\mathbf{E} \gamma$ при $v>\mathbf{E} \gamma($ см. (3.8)), то сравнение $(2.20)$ с (2.10) показывает, что в бесконечномерном случае вероятность попадания $\zeta_{n}$ в $B_{v}$ «экспоненциально больше», чем вероятность попадания 
в любое выпуклое множество $B \subset B_{v}$, снаружи касающееся множества $\Lambda_{v}$. В конечномерном случае различие между двумя названными вероятностями определялось степенным множителем (см. замечания к следствию 2.1).

3. Свойства случайной величины $\gamma=\Lambda(\xi)$ и ее функции уклонений.

3.1. Инвариантность величины $\gamma$ при линейном преобразовании над $\xi$. Известно (см., например, [4], [3]) что для фиксированных вектора $a \in \mathbf{R}^{d}$ и квадратной невырожденной матрицы $M$ справедливы соотношения

$$
\Lambda^{(\xi+a)}(\alpha)=\Lambda^{(\xi)}(\alpha-a), \quad \Lambda^{(\xi M)}(\alpha)=\Lambda^{(\xi)}\left(\alpha M^{-1}\right),
$$

где $M^{-1}$ - обратная к $M$ матрица. Поэтому

$$
\Lambda^{(\xi+a)}(\xi+a)=\Lambda^{(\xi)}(\xi+a-a)=\gamma,
$$

т.е. $\gamma$ при сдвиге $\xi$ не меняется. Аналогично находим

$$
\Lambda^{(\xi M)}(\xi M)=\Lambda^{(\xi)}\left(\xi M M^{-1}\right)=\gamma
$$

т.е. $\gamma$ при «повороте и сжатии» вектора $\xi$ также не меняется. Таким образом, линейное преобразование над вектором $\xi$ не меняет величину $\gamma$.

3.2. Свойства случайной величины $\gamma$ и функции $\Lambda^{(\gamma)}$ в одномерном случае $d=1$. Пусть $\mathrm{S}_{ \pm}$- границы множества $\Lambda_{<\infty}$ или, что то же, - границы выпуклого замыкания $\mathrm{S}$ носителя распределения величины $\xi, \Lambda_{ \pm}=\Lambda\left(\mathrm{S}_{ \pm}\right), \Lambda^{*}=\max \left\{\Lambda_{+}, \Lambda_{-}\right\}, \Lambda_{*}=\min \left\{\Lambda_{+}, \Lambda_{-}\right\}$.

Теорема 3.1. (i) Случайная величина $\gamma=\Lambda(\xi)$ удовлетворяет неравенствам

$$
\mathbf{P}(\gamma \geqslant v) \leqslant \begin{cases}2 e^{-v}, & \text { если } v \leqslant \Lambda_{*} \\ e^{-v}, & \text { если } v \in\left(\Lambda_{*}, \Lambda^{*}\right] ; \\ 0, & \text { если } v>\Lambda^{*} .\end{cases}
$$

(ii) Значение $\mathbf{E} \gamma$ удовлетворяет неравенству

$$
\mathbf{E} \gamma \leqslant 2-e^{-\Lambda_{*}}-e^{-\Lambda^{*}} \leqslant 2 .
$$

(iii) Имеет место альтернатива: либо $\Lambda^{*}=\infty$, и тогда

$$
\Lambda^{(\gamma)}(v) \sim v \quad n p u \quad v \rightarrow \infty
$$

(или, ито то же, $\left.\lambda_{+}^{(\gamma)}:=\sup \left\{t: \mathbf{E} e^{t \gamma}<\infty\right\}=1\right) ;$ либо $\Lambda^{*}<\infty$, и тогда в случае $\Lambda^{*}=\Lambda_{+}$выполняется $\gamma \leqslant \Lambda_{+}\left(\right.$и, следовательно, $\left.\lambda_{+}^{(\gamma)}=\infty\right)$, $\left.\mathbf{P}\left(\gamma=\Lambda_{+}\right)=-\ln \mathbf{P}\left(\xi=\mathrm{S}_{+}\right)>0\right) ;$ аналогичные соотношения справедливы в случае $\Lambda^{*}=\Lambda_{-}$. 
Д о к а з а т е л ь с т в о. (i) Рассмотрим уравнение

$$
\Lambda(\alpha)=v>0
$$

Если $v \leqslant \Lambda_{*}$, то в силу выпуклости функции $\Lambda(\alpha)$ и ее непрерывности изнутри $\left[\mathrm{S}_{-}, \mathrm{S}_{+}\right]$, существует два решения $\alpha_{ \pm}(v)$ этого уравнения; при этом $\alpha_{+}(v)>\mathbf{E} \xi, \alpha_{-}(v)<\mathbf{E} \xi$. Если $v \in\left(\Lambda_{*}, \Lambda^{*}\right]$, то существует одно решение $\alpha_{+}(v)$ (если $\Lambda_{*}=\Lambda_{-}$) или $\alpha_{-}(v)$ (если $\Lambda_{*}=\Lambda_{+}$). Второе решение мы положим равным $\mp \infty$ соответственно. Если $v>\Lambda^{*}$, то решений не существует, и мы положим $\alpha_{ \pm}(v)= \pm \infty$. Из сказанного следует, что событие $\{\Lambda(\xi) \geqslant v\}$ есть объединение двух непересекающихся событий $\left\{\xi \leqslant \alpha_{-}(v)\right\},\left\{\xi \geqslant \alpha_{+}(v)\right\}$ (эти события могут быть и пустыми). Поэтому для $\gamma=\Lambda(\xi)$ получаем

$$
\mathbf{P}(\gamma \geqslant v)=\mathbf{P}\left(\xi \leqslant \alpha_{-}(v)\right)+\mathbf{P}\left(\xi \geqslant \alpha_{+}(v)\right)
$$

так что в силу неравенства Чебышёва

$$
\mathbf{P}(\gamma \geqslant v) \leqslant e^{-\Lambda\left(\alpha_{-}(v)\right)}+e^{-\Lambda\left(\alpha_{+}(v)\right)} \leqslant \begin{cases}2 e^{-v}, & \text { если } v \leqslant \Lambda_{*} ; \\ e^{-v}, & \text { если } v \in\left(\Lambda_{*}, \Lambda^{*}\right] ; \\ 0, & \text { если } v>\Lambda^{*} .\end{cases}
$$

(ii) Второе утверждение вытекает из первого и равенства

$$
\mathbf{E} \gamma=\int_{0}^{\infty} \mathbf{P}(\gamma \geqslant v) d v
$$

(iii) Пусть $\Lambda^{*}=\infty$. Из (i) следует, что $\lambda_{+}^{(\gamma)} \geqslant 1$. Нам надо доказать обратное неравенство. Положим $\theta_{n}:=n^{-1} \sum_{k=1}^{n} \gamma_{k}$, где $\gamma_{k}$ - независимые копии $\gamma$. В силу теоремы 2.1

$$
\varlimsup_{n \rightarrow \infty} \frac{1}{n} \ln \mathbf{P}\left(\theta_{n} \geqslant v\right) \leqslant-\Lambda^{(\gamma)}(v) .
$$

Оценим снизу левую часть этого неравенства. В силу выпуклости функции $\Lambda$ для $\zeta_{n}:=n^{-1} \sum_{k=1}^{n} \xi_{k}$ выполняется

$$
\Lambda\left(\zeta_{n}\right) \leqslant \frac{1}{n} \sum_{k=1}^{n} \Lambda\left(\xi_{k}\right)=\theta_{n}
$$

и, следовательно,

$$
\mathbf{P}\left(\theta_{n} \geqslant v\right) \geqslant \mathbf{P}\left(\Lambda\left(\zeta_{n}\right) \geqslant v\right) \geqslant \mathbf{P}\left(\zeta_{n} \geqslant \alpha_{+}(v)\right) .
$$

Пусть для определенности $\Lambda_{+}=\infty$. Тогда функция $\Lambda(\alpha)$ непрерывно возрастает от 0 до $\infty$ на $(\mathbf{E} \xi, \infty)$, и, стало быть, $\Lambda\left(\alpha_{+}(v)+0\right)=$ 
$\Lambda\left(\alpha_{+}(v)\right)=v$. В силу принципа больших уклонений для $\zeta_{n}$ имеем

$$
\begin{aligned}
\varlimsup_{n \rightarrow \infty} \frac{1}{n} \ln \mathbf{P}\left(\theta_{n} \geqslant v\right) & \geqslant \varliminf_{n \rightarrow \infty} \frac{1}{n} \ln \mathbf{P}\left(\theta_{n} \geqslant v\right) \\
& \geqslant \varliminf_{n \rightarrow \infty} \frac{1}{n} \ln \mathbf{P}\left(\zeta_{n} \geqslant \alpha_{+}(v)\right) \\
& \geqslant-\Lambda\left(\alpha_{+}(v)+0\right)=-v .
\end{aligned}
$$

Из (3.2), (3.4) вытекает, что

$$
\Lambda^{(\gamma)}(v) \leqslant v
$$

Известно (см., например, [3]), что функция уклонений $\Lambda^{(\gamma)}(v)$ случайной величины $\gamma$ (как и любой другой случайной величины) допускает представление

$$
\Lambda^{(\gamma)}(v)=\int_{\mathbf{E} \gamma}^{v} \lambda^{(\gamma)}(u) d u
$$

где $\lambda^{(\gamma)}(u)$ - точка, в которой достигается верхняя грань в определении $\Lambda^{(\gamma)}(u)=\sup _{\lambda}\left\{\lambda u-\ln \mathbf{E} e^{\lambda \gamma}\right\}$ и при этом $\lambda^{(\gamma)}(u) \uparrow \lambda_{+}^{(\gamma)}:=\sup \left\{\lambda: \mathbf{E} e^{\lambda \gamma}<\right.$ $\infty\}$ при $u \uparrow \infty$. Отсюда следует, что существует

$$
\lim _{v \rightarrow \infty} \frac{\Lambda^{(\gamma)}(v)}{v}=\lambda_{+}^{(\gamma)}
$$

Из (3.5), (3.7) вытекает требуемое неравенство $\lambda_{+}^{(\gamma)} \leqslant 1$, означающее, что $\lambda_{+}^{(\gamma)}=1$.

Если $\Lambda^{*}<\infty$, то утверждение теоремы очевидным образом следует из (3.1) и равенства

$$
\Lambda_{+}=-\ln \mathbf{P}\left(\xi=\mathrm{S}_{+}\right)
$$

(см., например, [3]). Теорема 3.1 доказана.

Соотношение (3.7) и равенство $\lambda_{+}^{(\gamma)}=1$ означают, что в широких предположениях

$$
\mathbf{P}(\gamma \geqslant v)=e^{-v+o(v)} \quad \text { при } \quad v \rightarrow \infty
$$

(см. об этом также п. 3.3). Кроме того, из равенства $\lambda_{+}^{(\gamma)}=1$ в силу (3.6) вытекает неравенство

$$
\Lambda^{(\gamma)}(v)<v-\mathbf{E} \gamma \quad \text { для } \quad v>\mathbf{E} \gamma .
$$

П р и м е р 3.1. Пусть $\xi$ имеет нормальное распределение. В силу п. 3.1 можно считать, что $\mathbf{E} \xi=0, \mathbf{E} \xi^{2}=1$. Тогда $\Lambda(\alpha)=\alpha^{2} / 2$ (см., например, [3]), так что $\gamma=\xi^{2} / 2, \mathbf{E} \gamma=1 / 2$,

$$
\mathbf{P}(\gamma \geqslant v)=2 \mathbf{P}(\xi \geqslant \sqrt{2 v})=\frac{2}{\sqrt{2 \pi}} \int_{\sqrt{2 v}}^{\infty} e^{-u^{2} / 2} d u \sim \frac{1}{\sqrt{\pi v}} e^{-v} \quad \text { при } \quad v \rightarrow \infty .
$$


Далее,

$$
\mathbf{E} e^{\lambda \gamma}=\mathbf{E} e^{\lambda \xi^{2} / 2}=\frac{1}{\sqrt{2 \pi}} \int_{-\infty}^{\infty} e^{\lambda x^{2} / 2-x^{2} / 2} d x=\frac{1}{\sqrt{1-\lambda}} \quad \text { при } \quad \lambda \leqslant 1 .
$$

Поэтому уравнение для точки $\lambda^{(\gamma)}(v)$ принимает вид

$$
\left(\lambda v+\frac{1}{2} \ln (1-\lambda)\right)_{\lambda}^{\prime}=v-\frac{1}{2(1-\lambda)}=0
$$

и имеет единственное решение $\lambda^{(\gamma)}(v)=1-1 /(2 v)$. Отсюда находим

$$
\Lambda^{(\gamma)}(v)=\int_{\mathbf{E}_{\gamma}}^{v}\left(1-\frac{1}{2 u}\right) d u=v-\frac{1}{2}(\ln v+\ln 2+1) .
$$

Ясно также, что в рассматриваемом примере $\mathrm{S}_{ \pm}= \pm \infty, \Lambda_{ \pm}=\infty$.

П р и м е р 3.2. Пусть $\xi$ имеет показательное распределение. В силу п. 3.1 можно считать, что

$$
\mathbf{P}(\xi \geqslant t)=e^{-t} \quad \text { при } \quad t \geqslant 0 .
$$

Тогда (см., например, [3])

$$
\Lambda(\alpha)=\alpha-1-\ln \alpha, \quad \alpha \geqslant 0,
$$

так что $\gamma=\xi-1-\ln \xi$. Уравнение $\Lambda(\alpha)=v$ при $v \rightarrow \infty$ имеет решение $\alpha_{+}(v)=v+\ln v+1+O\left(v^{-1} \ln v\right)$. Поэтому

$$
\begin{aligned}
\mathbf{P}(\gamma \geqslant v) & =\mathbf{P}\left(\xi \geqslant v+\ln v+1+O\left(v^{-1} \ln v\right)\right) \\
& =e^{-\left(v+\ln v+1+O\left(v^{-1} \ln v\right)\right)}=v^{-1} e^{-v-1}\left(1+O\left(v^{-1} \ln v\right)\right) \quad \text { при } \quad v \rightarrow \infty .
\end{aligned}
$$

В этом примере $\mathrm{S}_{-}=0, \mathrm{~S}_{+}=\infty, \Lambda_{ \pm}=\infty$.

П р и м е р 3.3. Для бернуллиевской случайной величины $\xi, \mathbf{P}(\xi=$ $1)=p=1-\mathbf{P}(\xi=0)$, выполняется (см., например, [3])

$\gamma=\Lambda(\xi)=\xi \ln \frac{\xi}{p}+(1-\xi) \ln \frac{1-\xi}{1-p}, \quad \mathbf{E} \gamma=-p \ln p-(1-p) \ln (1-p) \leqslant \ln 2$.

Поэтому

$$
\gamma= \begin{cases}-\ln p & \text { с вероятностью } p, \\ -\ln (1-p) & \text { с вероятностью } 1-p .\end{cases}
$$

При $p=1 / 2$ величина $\gamma$ вырождается в постоянную $\ln 2$. Если $p<1 / 2$, то $\gamma$ есть линейное преобразование над $\xi$ :

$$
\gamma=\frac{\xi+a}{b-a} \quad \text { при } \quad a=\ln (1-p), \quad b=\ln (1-p)-\ln p,
$$

так что

$$
\Lambda^{(\gamma)}(v)=\Lambda(v(b-a)-a) .
$$

Случай $p>1 / 2$ рассматривается аналогично. В этом примере $\mathrm{S}_{-}=0$, $\mathrm{S}_{+}=1, \Lambda_{-}=-\ln (1-p), \Lambda_{+}=-\ln p$. 
3.3. Свойства случайной величины $\gamma$ и функции $\Lambda^{(\gamma)}$ в случае $d>1$. В многомерном случае $d>1$ анализ свойств случайной величины $\gamma$ оказывается более сложным. Мы ограничимся рассмотрением векторов $\xi$, распределение которых на бесконечности ведет себя достаточно правильно.

О п р е д е л е н и е 1 . Мы будем говорить, что одномерная случайная величина $\xi$ принадлежит классу $\mathscr{L}$, если

$$
\ln \mathbf{P}(\xi \geqslant t) \sim-\Lambda^{(\xi)}(t) \quad \text { при } \quad t \rightarrow \infty .
$$

Соотношение (3.9) есть соотношение того же типа, что и в принципе больших уклонений, но при $n=1, t \rightarrow \infty$.

Класс $\mathscr{L}$ достаточно широк и содержит в себе классы $\mathscr{E} \mathscr{R}, \mathscr{E} \mathscr{S}, \mathscr{S} \mathscr{E}$ (см. [13]) случайных величин $\xi$, для которых соответственно

$$
\mathbf{P}(\xi \geqslant t)=e^{-\lambda_{+} t} t^{\alpha} L(t), \quad \mathbf{P}(\xi \geqslant t)=e^{-\lambda_{+} t \pm t^{\beta} L(t)}, \quad \mathbf{P}(\xi \geqslant t)=e^{-t^{\nu} L(t)},
$$

где $\lambda_{+}:=\sup \left\{\lambda: \mathbf{E} e^{\lambda \xi}<\infty\right\}>0, \alpha \in(-\infty, \infty), \beta \in(0,1), \nu>2, L(t)-$ медленно меняющаяся на бесконечности функция.

В качестве гипотезы можно назвать следующий критерий принадлежности величины $\xi$ классу $\mathscr{L}$ : для того чтобы случайная величина $\xi$ принадлежала классу $\mathscr{L}$, необходимо и достаточно, итобь существовала выпуклая вниз функиия $H(t)$ такая, что $-\ln \mathbf{P}(\xi \geqslant t) \sim H(t)$ при $t \rightarrow \infty$. Если такая функиия существует, то $H(t) \sim \Lambda(t)$ при $t \rightarrow \infty$.

Ниже будем предполагать, что $\Lambda_{<\infty}=\mathbf{R}^{d}$. Тогда множества $\Lambda_{v}$ неограниченно расширяются при $v \rightarrow \infty$. Обозначим $B_{v}:=\{\alpha: \Lambda(\alpha) \geqslant$ $v\}, \Lambda_{v-}:=\{\alpha: \Lambda(\alpha)<v\}$ и положим $\Pi(\mathrm{e}, b):=\{\alpha:\langle\mathrm{e}, \alpha\rangle \geqslant b\}$. Нам понадобится следующее условие.

[П]. Для любого $\varepsilon>0$ существует конечное число $K_{\varepsilon}$ полупространств $\Pi\left(\mathrm{e}_{k, v}, b_{k, v}\right)$ таких, что при всех достаточно больших $v$ вы-полняется

1) $B_{v} \subset U_{v}:=\bigcup_{k} \Pi\left(\mathrm{e}_{k, v}, b_{k, v}\right)$;

2) $\Lambda_{v(1-\varepsilon)-} \cap U_{v}=\varnothing\left(\right.$ можно считать, ито $\Pi\left(\mathrm{e}_{k, v}, b_{k, v}\right)$ касаются множества $\left.\Lambda_{v(1-\varepsilon)}\right)$.

Условие [П] означает, что множества $\Lambda_{v(1-\varepsilon)}$ и $B_{v}$ могут быть разделены с помощью многогранника, имеющего $K_{\varepsilon}$ плоских граней.

Условие [П] выполнено, например, для класса случайных векторов $\xi$, для которых выполнено условие $\left[\mathbf{C}_{0}\right]$, а множество $A_{<\infty}:=\{\lambda: A(\lambda)<\infty\}$ ограничено в $\mathbf{R}^{d}$. Действительно, для таких векторов функция уклонений $\Lambda$ асимптотически линейна по любому направлению е:

$$
\Lambda(t \mathrm{e}) \sim t \Lambda_{+}(\mathrm{e}) \text { при } t \rightarrow \infty
$$

где $\Lambda_{+}(\mathrm{e}):=\sup _{\lambda \in A_{<\infty}}\langle\lambda, \mathrm{e}\rangle$ (см. [13]). Поэтому поверхности $\partial \Lambda_{v}$ уровня $v$ функции $\Lambda(\alpha)$ являются «асимптотически концентрическими поверхностями», которые после сжатия в $v$ раз сближаются с поверхностью 
$\Gamma_{1}:=\left\{\alpha: \Lambda_{+}(\alpha)=1\right\}$ при $v \rightarrow \infty$, а поверхность $\partial \Lambda_{v(1-\varepsilon)}$ уровня $v(1-\varepsilon)$ сближается с поверхностью $\Gamma_{1-\varepsilon}:=\left\{\alpha: \Lambda_{+}(\alpha)=(1-\varepsilon)\right\}$. Так как выполнено условие $\left[\mathbf{C}_{0}\right]$, то $\inf _{\mathrm{e}} \Lambda_{+}(\mathrm{e})>0$ и поверхности $\Gamma_{1}, \Gamma_{1-\varepsilon}$ не соприкасаются. Кроме того, они являются границами выпуклых множеств. Поэтому ясно, что между $\Gamma_{1}$ и $\Gamma_{1-\varepsilon}$ существует разделяющий многогранник и условие [П] выполнено.

Условие ограниченности множества $A_{<\infty}$ для выполнения [П] не является существенным. Пусть, например, $\Lambda(\alpha)$ растет при $|\alpha| \rightarrow \infty$ квадратичным образом с точностью до слагаемого $o\left(|\alpha|^{2}\right)$ (в этом случае $A_{<\infty}=\mathbf{R}^{d}$ ). Тогда, сделав нужное линейное преобразование, мы получим функцию $\sum\left|\alpha_{i}\right|^{2}+o\left(|\alpha|^{2}\right)$, для которой поверхности уровня $v$ будут сближаться после сжатия в $\sqrt{v}$ раз со сферой $\sum\left|\alpha_{i}\right|^{2}=1$, а поверхности уровня $v(1-\varepsilon)-$ со сферой $\sum\left|\alpha_{i}\right|^{2}=1-\varepsilon$. Так как многогранники при линейном преобразовании переходят в многогранники, то свойство [П] здесь, очевидно, выполнено. Учитывая замечание 3.1 (см. ниже), можно рассматривать и другие виды сходимости $\Lambda(\alpha)$ к $\infty$ при $|\alpha| \rightarrow \infty$.

Теорема 3.2. Пусть $\Lambda_{<\infty}=\mathbf{R}^{d}$ и выполнено условие [П]. Тогда:

(i) $\lambda_{+}^{(\gamma)}=1, \Lambda^{(\gamma)}(v)=v+o(v) n p u v \rightarrow \infty$;

(ii) если дополнительно найдется единичный вектор е такой, что $\langle\mathrm{e}, \xi\rangle \in \mathscr{L}$, mo nрu $v \rightarrow \infty$

$$
\mathbf{P}(\gamma \geqslant v)=e^{-v+o(v)} \quad u \quad \gamma \in \mathscr{L} .
$$

Д о к а з а те ль с т в о. (i) Оценка сверху. Имеем в силу теоремы 1.2

$$
\begin{aligned}
\mathbf{P}(\gamma \geqslant v) & =\mathbf{P}\left(\xi \in B_{v}\right) \leqslant \mathbf{P}\left(\xi \in \bigcup_{1 \leqslant k \leqslant K_{\varepsilon}} \Pi\left(\mathrm{e}_{k, v}, b_{k, v}\right)\right) \\
& \leqslant \sum_{k=1}^{K_{\varepsilon}} \mathbf{P}\left(\xi \in \Pi\left(\mathrm{e}_{k, v}, b_{k, v}\right)\right) \leqslant \sum_{k=1}^{K_{\varepsilon}} e^{-\Lambda\left(\Pi\left(\mathrm{e}_{k, v}, b_{k, v}\right)\right)} \leqslant K_{\varepsilon} e^{-v(1-\varepsilon)} .
\end{aligned}
$$

Стало быть,

$$
\ln \mathbf{P}(\gamma \geqslant v) \leqslant-(1-\varepsilon) v+\ln K_{\varepsilon} .
$$

Так как $\varepsilon>0$ произвольно, то

$$
\varlimsup_{v \rightarrow \infty} \frac{1}{v} \ln \mathbf{P}(\gamma \geqslant v) \leqslant-1, \quad \lambda_{+}^{(\gamma)} \geqslant 1 .
$$

Оиенка снизу. Воспользуемся соотношениями (3.2), (3.3), из которых следует, что

$$
-\Lambda^{(\gamma)}(v) \geqslant \varlimsup_{n \rightarrow \infty} \frac{1}{n} \ln \mathbf{P}\left(\Lambda\left(\zeta_{n}\right) \geqslant v\right)=\varlimsup_{n \rightarrow \infty} \frac{1}{n} \ln \mathbf{P}\left(\zeta_{n} \in B_{v}\right) .
$$


Так как $\Lambda_{<\infty}=\mathbf{R}^{d}$, то функция $\Lambda(\alpha)$ всюду непрерывна и, следовательно, $\Lambda\left(\left(B_{v}\right)\right)=\Lambda\left(B_{v}\right)=v$. Поэтому в силу принципа больших уклонений для нормированных сумм $\zeta_{n}=n^{-1} \sum_{k=1}^{n} \xi_{k}$ выполняется

$-\Lambda^{(\gamma)}(v) \geqslant \varlimsup_{n \rightarrow \infty} \frac{1}{n} \ln \mathbf{P}\left(\zeta_{n} \in B_{v}\right) \geqslant \varliminf_{n \rightarrow \infty} \frac{1}{n} \ln \mathbf{P}\left(\zeta_{n} \in B_{v}\right) \geqslant-\Lambda\left(\left(B_{v}\right)\right)=-v$.

Мы получили, что $\Lambda^{(\gamma)}(v) \leqslant v, \lambda_{+}^{(\gamma)} \leqslant 1$. Вместе с (3.10) это доказывает первое утверждение теоремы.

(ii) Из (3.10) вытекает, что

$$
\mathbf{P}(\gamma \geqslant v) \leqslant e^{-v+o(v)} \quad \text { при } \quad v \rightarrow \infty .
$$

Поэтому для доказательства второго утверждения нам достаточно установить обратное неравенство.

Для любого $v>0$ и любого фиксированного е имеем

$$
\mathbf{P}(\gamma \geqslant v)=\mathbf{P}\left(\xi \in B_{v}\right) \geqslant \mathbf{P}\left(\xi \in \Pi\left(\mathrm{e}, b_{v}\right)\right)=\mathbf{P}\left(\langle\mathrm{e}, \xi\rangle \geqslant b_{v}\right),
$$

где $b_{v}$ выбрано так, что полупространство П $\left(\mathrm{e}, b_{v}\right)$ касается $\partial B_{v}$. Как и в $\S 1$, убеждаемся, что $\Lambda^{(\langle\mathrm{e}, \xi\rangle)}\left(b_{v}\right)=v$. Ясно, что $b_{v} \rightarrow \infty$ при $v \rightarrow \infty$. Выберем е таким образом, что $\langle$ е, $\xi\rangle \in \mathscr{L}$. Тогда при $v \rightarrow \infty$

$$
\ln \mathbf{P}(\gamma \geqslant v) \geqslant \ln \mathbf{P}\left(\langle\mathrm{e}, \xi\rangle \geqslant b_{v}\right) \sim-\Lambda^{(\langle\mathrm{e}, \xi\rangle)}\left(b_{v}\right)=-v .
$$

Это означает, что

$$
\mathbf{P}(\gamma \geqslant v) \geqslant e^{-v+o(v)} \quad \text { при } \quad v \rightarrow \infty .
$$

Теорема доказана.

3 а м е ч а н и е 3.1. (i) Из доказательства теоремы 3.2 видно, что утверждение этой теоремы останется справедливым и в том случае, когда число $K_{\varepsilon}$ полупространств в условии $[\Pi]$ растет вместе с $v$, но так, что $\ln K_{\varepsilon}=o(v)$ при $v \rightarrow \infty$.

(ii) Если $\Lambda_{<\infty}$ не совпадает с $\mathbf{R}^{d}, d \geqslant 2$, то случайная величина $\gamma=\Lambda(\xi)$ может и не удовлетворять условию Крамера $\left[\mathbf{C}_{0}\right]$, о чем свидетельствует пример 1.2, рассмотренный в 1 . Напомним, что в примере 1.2 случайный вектор $\xi$ имеет равномерное распределение на единичной сфере $\Gamma$ в $\mathbf{R}^{d}, d \geqslant 2$. Поскольку для любого $\alpha \in \Gamma$ выполняется $\Lambda(\alpha)=\infty$, то $\gamma=\Lambda(\xi)=\infty$ с вероятностью 1 , и условие Крамера для $\gamma$ не выполнено.

П р и м е р 3.4. Пусть вектор $\xi=\left(\xi_{(1)}, \ldots, \xi_{(d)}\right)$ имеет нормальное распределение с нулевым средним и единичной ковариационной матрицей. Тогда функция уклонений имеет вид (см., например, [3]) $\Lambda(\alpha)=$ $(1 / 2) \sum_{i=1}^{d} \alpha_{i}^{2}$ и случайная величина

$$
2 \gamma=2 \Lambda(\xi)=\xi_{(1)}^{2}+\cdots+\xi_{(d)}^{2}
$$


имеет известное распределение $\chi^{2}$ с $d$ степенями свободы, так что

$$
\mathbf{P}(\gamma \geqslant v)=\frac{1}{\Gamma(d / 2)} \int_{v}^{\infty} u^{d / 2-1} e^{-u} d u \sim \frac{1}{\Gamma(d / 2)} v^{d / 2-1} e^{-v} \quad \text { при } \quad v \rightarrow \infty .
$$

Условия теоремы 3.2 здесь очевидно выполнены. При $d=2$ распределение $\gamma$ оказывается в точности экспоненциальным: $\mathbf{P}(\gamma \geqslant v)=e^{-v}$, $v \geqslant 0$.

Заметим также, что если координаты $\xi_{(i)}$ вектора $\xi$ независимы, то $\Lambda(\alpha)=\sum_{i=1}^{d} \Lambda^{\left(\xi_{(i)}\right)}\left(\alpha_{i}\right)$, и изучение свойств $\gamma$ и $\Lambda^{(\gamma)}(v)$ во многом сводится к одномерному случаю, так как к независимым слагаемым $\Lambda^{\left(\xi_{(i)}\right)}\left(\xi_{(i)}\right)$ применима теорема 3.1. В частности, всегда имеет место неравенство $\mathbf{E} \gamma \leqslant 2 d$.

\section{СПИСОК ЛИТЕРАТУРЫ}

1. Боровков A.A., Могульский A.A. О принципах больших уклонений в метрических пространствах. - Сиб. матем. журн., 2010, т. 51, № 6, с. 1251-1269.

2. Боровков A.A., Могульский A.А. Расширенный принцип больших уклонений для траекторий случайных блужданий. I, II. Препринт. Новосибирск: ИМ СО PAH, 2010.

3. Боровков A. А.. Теория вероятностей. М.: Эдиториал УРСС, 2009, 652 с.

4. Боровков A.А., Могульский A.А. Большие уклонения и проверка статистических гипотез. Новосибирск: Наука, 1992, 222 с.

5. Функциональный анализ. М.: Наука, 1964, 424 с. (Справочная математическая библиотека.)

6. Рокафеллар Р. Выпуклый анализ. М.: Мир, 1973, 469 с.

7. Колмогоров A.H., Фомин С.В. Элементы теории функций и функционального анализа. М.: Наука, 1968, 496 с.

8. Боровков A. А. Некоторые неравенства для сумм многомерных случайных величин. - Теория вероятн. и ее примен., 1968, т. 13, в. 1, с. 255-159.

9. Боровков A.A. Граничные задачи для случайных блужданий и большие уклонения в функциональных пространствах. - Теория вероятн. и ее примен., 1967, т. 12 , в. 4 , с. $635-654$.

10. Могульский A. А. Большие уклонения для траекторий многомерных случайных блужданий. - Теория вероятн. и ее примен., 1976, т. 21, в. 2, с. 309-323.

11. Varadhan S. R. S. Asymptotic probabilities and differential equations. - Comm. Pure Appl. Math., 1966, v. 19, № 3, p. 261-286.

12. Боровков A.A., Могульский A.A. Свойства функционала уклонений от траекторий, возникающего при анализе вероятностей больших уклонений случайных блужданий. Препринт. Новосибирск: ИМ СО РАН, 2010.

13. Боровков A. А., Могульский A. А. О больших и сверхбольших уклонениях сумм независимых случайных векторов при выполнении условия Крамера. I, II. - Teория вероятн. и ее примен., 2006, т. 51, в. 2, с. 260-294; в. 4, с. 641-673.

Поступила в редакцию 20.X.2010 